Current draft: June 10, 2022

First draft: February 1, 2012

\title{
An Empirical Assessment of Characteristics and Optimal Portfolios
}

\author{
Christopher G. Lamoureux* \\ and
}

Huacheng Zhang ${ }^{\dagger}$

\begin{abstract}
We show that overfitting plagues optimal portfolios obtained by linking weights directly to characteristics. We effectively mitigate overfitting (regularize) by optimizing a more concave loss function than the investor's utility function. Significant certainty equivalent gains over benchmarks require at least three characteristics: momentum, size, and residual volatility. Out-of-sample utility gains are due to characteristic complementarities, and depend on investor risk aversion. For example, conditioning on momentum relieves the overfitting bias inherent in the other characteristics for our most risk-averse investor. Optimal portfolios' returns lie largely outside the span of traditional factors and move closer to the market as risk-aversion increases.
\end{abstract}

Key Words: cross-section of stock returns; overfitting; stock characteristics; optimal portfolios; out-of-sample evaluation

* Department of Finance, The University of Arizona, Eller College of Management, Tucson, 85721, 520-6217488, lamoureu@arizona.edu.

${ }^{\dagger}$ Department of Finance, Risk, and Banking, Nottingham University Business School, UK, zhanghuacheng1@gmail.com.

The current version of this paper may be downloaded from lamfin.arizona.edu/rsch.html . 


\section{Introduction}

Brandt, Santa-Clara, and Valkanov (2009) introduce a brilliant algorithm to optimize an insample utility function by linking portfolio weights directly to measurable characteristics. They demonstrate that characteristics can be used by power utility investors to build portfolios that have a higher certainty equivalent than standard benchmarks. This approach eliminates the need to specify a data generating process and estimate conditional moments of the return distribution, traditionally the first step in portfolio optimization. They also show that these optimized portfolios' relative performance degrades out of sample. We are motivated to expand the vast literature that has looked at characteristics' predictive content beyond the mean-variance framework using this algorithm. In order to do that we first have to document, understand, and manage the algorithm's estimation risk, which is known to plague optimal portfolio selection. That is, we have no interest in the properties of in-sample optimal portfolios if these portfolios have poor out-of-sample performance.

This paper's first contribution is to extend Brandt, Santa-Clara, and Valkanov (2009) to analyze estimation risk in the context of power utility investors with varying degrees of relative risk aversion. We find that overfitting has a first-order effect on portfolios selected using the algorithm, as is the case with mean-variance optimization. For most characteristic combinations the optimal portfolios do not dominate market benchmarks out of sample as a result of overfitting. This overfitting problem arises despite the small number of estimated coefficients as a result of the dimensionality of the characteristic space and the infinite dimensionality of the parameter space, as the utility function depends on all of the properties of the unspecified multivariate conditional return distribution. Our first major result is that increasing the curvature of the loss function used to obtain portfolio weights relative to the utility function preserves benefits from conditioning on characteristics, and mitigates overfitting.

In-sample optimal portfolios perform notoriously poorly out of sample. Most of the work done to understand and mitigate this problem is in the context of mean-variance optimization. Best and Grauer (1991) stress that this deterioration results from optimization amplifying estimation errors. The models that have been developed to manage estimation risk include constraining weights and constraining estimators ${ }^{1}$ However, DeMiguel, Garlappi, and Uppal (2009) show that in general, these economic and statistical tools that have been developed to optimize in the

\footnotetext{
${ }^{1}$ Jagannathan and Ma (2003) demonstrate that there is a duality between constraining the weights and shrinking moment estimators in the mean-variance optimization context. Kan and Zhou (2007) derive the expected loss function from using sample (rather than true) moments when returns are normally distributed. They show that estimation risk can be diversified by holding a minimum variance portfolio in addition to the estimated tangency portfolio. Recent Bayesian approaches establish a prior using economic theory. Pástor (2000) and Pástor and Stambaugh (2000) use asset pricing models to form the prior. MacKinlay and Pástor (2000) impose moment restrictions according to a factor model.
} 
presence of estimation error are dominated by the $\frac{1}{N}$ rule.

Part of the motivation for linking portfolio weights to characteristics is that portfolio selection is reduced to a single step. Traditionally portfolio choice is split into two steps (Gennotte 1986), with the first step being estimation of the conditional return distribution. In the mean-variance context this entails obtaining consistent estimators of the conditional mean and variance covariance matrix. Aït-Sahalia and Brandt (2001, p. 1299) refer to conditional moment (or conditional likelihood) estimation as the "Achilles' heel of conditional portfolio choice because although the moments are predictable, this predictability is for some moments quite tenuous." They argue that by not specifying a likelihood (i.e., the conditional return distribution) we avoid "introducing additional noise and potential misspecifications through the intermediate, but unnecessary, estimation of the return distribution." Brandt, Santa-Clara, and Valkanov (2009, p. 3412) say that linking optimized weights directly to characteristics "escapes the common statistical problems of imprecise coefficient estimates and overfitting."

Brandt, Santa-Clara, and Valkanov (2009, p. 3423) note that shrinkage is a common approach to mitigating overfitting. Shrinkage via regularization generally entails an auxiliary loss function with separate tuning parameters, such as with a Lasso (L1-norm) penalty on the estimated coefficients. The regularized loss function is maximized over the tuning parameters in a (cross-) validation step. DeMiguel, Martín-Utrera, Nogales, and Uppal (2020) use the direct optimization algorithm to maximize the Sharpe ratio (i.e., they specify a quadratic utility function), with transactions costs. They impose an L1-norm penalty, and demonstrate that it does better out of sample than a non-regularized optimization. However, Kozak, Nagel, and Santosh (2020, p.274) note that a Lasso-type L1-norm penalty has poor statistical properties when the characteristics are correlated, and it lacks economic motivation.

Brandt, Santa-Clara, and Valkanov (2009) show that as investor risk aversion increases the coefficients on their three characteristics tend to decrease in absolute value. However, these effects are not uniform across the characteristics, and there are sign changes. This suggests an alternative to shrinkage which we implement. Rather than introduce a statistical penalty on the sizes of the coefficients managed with a tuning parameter, we treat the concavity of the loss function used on the sample as the tuning parameter, disentangling the loss function optimized on the data from the investor's utility function. For example consider a power utility investor with coefficient of relative risk aversion, $\gamma=2$. For this loss function, we optimize in sample using power "utility" functions indexed by $\gamma^{*}=2,3,4, \ldots$, and evaluate the sampling distribution of the certainty equivalent returns. The characteristic set comprising: momentum, log size, beta, residual volatility, and average same-month return is optimal for this loss function. Using this set and $\gamma^{*}=\gamma=2$ generates an optimal (in-sample) portfolio whose mean certainty equivalent 
return over the 528-month out-of-sample period is $-42 \%$ per month. By contrast, when $\gamma^{*}=3$, this statistic is $3 \%$ per month. We find similar results for all levels of relative risk aversion. Using $\gamma^{*}=\gamma=5$ with the optimal characteristic set generates an optimal portfolio with a $95 \%$ certainty equivalent confidence interval of $(50,146)$ basis points per month, which does not statistically dominate the benchmark. The comparable values for the optimal portfolio when $\gamma=5$ and $\gamma^{*}=10$, in this case are $(133,170)$ basis points per month.

This method of regularization, or shrinkage, affords a natural interpretation of the tuning parameter, $\gamma^{*}$. Relative to $\gamma$, it measures the increase in the costs of even moments in terms of odd moments needed to offset overfitting. It also allows the degree of shrinkage to vary across loss functions and characteristic sets, as well as allowing for sign changes. And unlike the Lasso penalty its efficacy is not affected by high correlations among the factors. The effectiveness of this method to mitigate overfitting is an empirical question. If overfitting results from apparent spurious near-arbitrage opportunities in the sample then our regularization of increasing the concavity of the loss function used on the data would be ineffective. We show that this is not the case. That is, the spurious sample patterns are the result of outliers-largely exaggerations of a stationary data generating process. Consequently, making even moments more costly in terms of odd moments produces superior out-of-sample results.

Our out-of-sample model assessment is more robust to data mining and less sensitive to outliers than in-sample inference. Because we optimize in-sample and evaluate out-of-sample we use a long time series-our data start in 1955 and end in 2018. We contrast a rolling sample optimization design with an updating design to ascertain whether there have been structural breaks in the relationships between characteristics and returns over our sample period. We find that the rolling design is subject to more severe overfitting-requiring a bigger adjustment of $\gamma^{*}$ relative to $\gamma$ to regulate. This has the effect of reducing the beneficial informational content of the characteristics, and therefore the rolling design is never preferred to updating. The fact that updating serves to mitigate overfitting is further evidence that the overfitting results from "outliers," and manifests largely in exaggerated patterns rather than spurious arbitrage opportunities.

We extend the large literature on cross-sectional return predictability beyond the meanvariance framework. Since power utility is a function of all of the moments of the return distribution we extend analysis of characteristics' predictive content to higher moments including skewness and kurtosis. Leland (1999), Ferson (2013), Broadie, Chernov, and Johannes (2009), and Goetzmann, Ingersoll, Spiegel, and Welch (2007) raise concerns about measuring performance using linear measures such as the Sharpe ratio, or alpha from a factor model when the return process is non-Gaussian. Harvey and Siddique (2000) argue that conditional skewness in stock returns can explain deviations from linear factor pricing models. Furthermore, Patton (2004) shows 
that there is enough persistence in higher-order moments to generate significant utility gains to a constant relative risk aversion (CRRA) investor in an asset allocation problem. Motivating our extention of portfolio performance beyond the first two moments, Barroso and Santa-Clara (2015) and Kadan and Liu (2014) show that characteristic-based portfolios that generate a high alpha and Sharpe ratio come at the cost of negative skewness.

Much of the recent research on characteristics' predictive content for cross-sectional stock returns raises data mining concerns. Giglio, Liao, and Xiu (2021), Hou, Xue, and Zhang (2020), Harvey, Liu, and Zhu (2016), and Harvey (2017) stress the multiple test nature of testing characteristics' predictive efficacy. Researchers have scoured the same data in an attempt to identify characteristics to predict means, after accounting for known sources of systematic variance. Another statistical concern is the temporal nature of findings of predictability. Linnainmaa and Roberts (2018) show that most accounting-based anomalies are not robust to out-of-sample testing. McLean and Pontiff (2016) argue that characteristics' predictive content vanishes once the relationship is published in our academic literature. On the theoretical front, Martin and Nagel (2020) model agents' learning about cross-sectional predictive relationships. In this model there is evidence of in-sample predictability which is not present out of sample. We address data mining on two fronts. First we use a different loss function than that used in most earlier studies. As noted above, even predictive content that is robust to data mining concerns of alpha or the Sharpe ratio may not be useful to a CRRA utility function. Our resampled out-of-sample design addresses the temporality concerns, including learning. Finally the effects of overfitting are also loss function dependent. We compare models, evaluate predictive efficacy and characteristics' roles using confidence intervals on optimal portfolio out-of-sample certainty equivalent returns.

We evaluate sampling distributions of the out-of-sample certainty equivalent returns for power utility investors with varying degrees of risk aversion across 48 different sets of characteristics. We measure the marginal economic effect of each characteristic on the small sample distribution of the certainty equivalent in the context of the complementary characteristics (Cochrane 2011). We show that no single characteristic can be used by all power utility investors to outperform the relevant market benchmark $2^{2}$ Our second major result is that the gains from linking portfolio weights to measurable characteristics derive mostly from complementarities amongst the characteristics as they relate to the cross-section of future returns. All power utility investors can outperform the benchmarks using the characteristic triplet: momentum, log size, and residual volatility. As risk tolerance rises, average same-month return is added to the optimal characteristic set, and

\footnotetext{
${ }^{2}$ As a matter of exposition, we will use the phrase "Portfolio A outperforms the benchmark" to mean that the $2.5 \%$ ile of the sampling distribution of portfolio A's certainty equivalent return exceeds the $97.5 \%$ ile of the sampling distribution of the benchmark portfolio's certainty equivalent return. The "market benchmark" refers to either the equally-weighted portfolio of all stocks or the value-weighted portfolio of all sample stocks, depending on which the investor prefers.
} 
the most risk-tolerant investors we consider also include beta. We find that the book-to-market ratio is never part of an optimal characteristic set because it is redundant in the context of size and residual volatility. In their mean-variance setting, DeMiguel, Martín-Utrera, Nogales, and Uppal (2020) show that combining characteristics has a salutary trading-diversification effect. This characteristic complementarity is very different from the forms we find. We document that the predictive density of a small stock with positive momentum, for example, is very different from that of a small stock with negative momentum.

Since much of the literature on identifying predictive characteristics seeks to identify the incremental power of a factor conditional on another set of factors, complementarity amongst highly correlated characteristics has been largely ignored. For example, Feng, Giglio, and Xiu (2020) note that the month-of-the-year factor (Heston and Sadka 2008) has a significant alpha benchmarked to the Fama-French 3-factor model. However, since it has a correlation of 0.63 with the momentum factor, including momentum in the benchmarking model renders the portfolio alpha statistically insignificant. We show that for a power utility investor with $\gamma=2$, adding average same-month return to the characteristic set: size, beta, residual volatility, and momentum, leads to a statistically significant increase in the out-of-sample certainty equivalent return. Conversely, if this investor excludes momentum, and allows weights to depend on size, beta, residual volatility, and average same-month return, the resulting optimal portfolio suffers from severe overfitting and fails to dominate the benchmark out of sample. In contrast, a power utility investor with $\gamma=9$, derives no incremental benefits from including average same-month return in the set of characteristics used to tilt weights away from the market portfolio.

Our third major result demonstrates how and why characteristics are used to construct preferred portfolios and how risk aversion interacts with characteristic use. Our power utility investors use characteristics both to achieve some exposure to the Fama-French-Carhart (FFC) factors, and to shift away from the market. For example, the optimal portfolio for the power utility investor with $\gamma=5$ has a market beta of 0.6 , and only $3 \%$ of the portfolio's variance comes from the market variance. This portfolio has a loading of 1.0 on the size factor (SMB), and derives $5 \%$ of its variance from this source. By contrast, these values for the benchmark (equally-weighted portfolio) are 0.5 and $8 \%$, respectively. Similarly the optimal portfolio's loading on the value factor (HML) is 2.0, with $17 \%$ variance attribution. The benchmark's HML exposures are 0.3 and $2 \%$. The momentum factor (MOM) accounts for $20 \%$ of the optimal portfolio's variance, as this portfolio's MOM loading is 1.5. The equally-weighted benchmark has a momentum loading of -0.1 , and it derives less than $1 \%$ of its variance from this factor. Finally, this portfolio's alpha is 269 basis points per month, and $69 \%$ of the portfolio variance lies outside the span of the four factors. 
Market beta is flat in $\gamma$, so that the percent of portfolio variance attributed to the market factor increases in risk aversion. By contrast, the optimal portfolio's loading on the momentum factor decreases in risk aversion, whereas the percent of variance attributed to this factor is constant across $\gamma$. The loading on SMB and relative importance of this factor monotonically decrease in $\gamma$. The optimal portfolio for the power utility investor with $\gamma=2$ has a significantly positive SMB loading, the optimal portfolio for the investor with $\gamma=5$ has an insignificant SMB loading, and the loading on SMB of the optimal portfolio for the investor with $\gamma=9$ is significantly negative. The value factor's appeal increases in risk aversion. It accounts for $37 \%$ of the portfolio variance of the optimal portfolio of the investor with $\gamma=9$, and $20 \%$ of the optimal portfolio of the investor with $\gamma=2$. The four factors account for $32 \%, 42 \%$, and $54 \%$ of optimal portfolio variance as $\gamma$ increases from 2 to 5 to 9 .

These decompositions shed further light on the characteristic complementarity. For the power utility investor with $\gamma=2$, adding average same-month return to the characteristics: momentum, size, beta, and residual volatility significantly reduces the portfolio's exposure to the momentum factor, with no change in exposure to the other three factors.

Figure 1 shows the out-of-sample return distribution of the optimal characteristic-based portfolio for the power utility investor with $\gamma=9$ and the value-weighted (market) portfolio. The optimal portfolio is tilted toward stocks with positive momentum, smaller market capitalizations, and lower residual volatility (and away from stocks at the other end of the spectrum along these dimensions). The optimal portfolio is symmetric, whereas the benchmark has a negative skew. The optimal portfolio is significantly more leptokurtotic than the market. The optimal portfolio's mean return is 176 basis points per month, the market's mean return is 106 basis points per month. The optimal portfolio's return interquartile range of 4.6 (percent per month) is significantly less than the benchmark's $5.2 \%$. However the optimal portfolio's return standard deviation $(4.5 \%)$ is not significantly different from the benchmark's $4.3 \%$. This lack of consistency in the comparisons of measures of the distributions' scales highlights the importance of not assuming that portfolio returns are normally distributed. In general, the standard deviation is not a good measure of scale for a skewed and/or leptokurtotic distribution. The mean (95\%ile confidence interval) of the optimal portfolio's certainty equivalent return (to this investor) is $62(44,77)$ basis points per month. These statistics for the benchmark are $10(4,15)$.

This complementarity of correlated characteristics together with the positioning of portfolios outside the span of the Fama-French-Carhart factors, our management of estimation risk, and the behavior of portfolios as risk aversion increases are all consistent with the arguments in Kozak, Nagel, and Santosh (2018) and the findings of Kozak, Nagel, and Santosh (2020), who only consider the first two moments of the return distribution. A multivariate set of correlated 
characteristics identifies tradeoffs in the data of odd for even moments. A relatively risk tolerant investor wants exposure to a factor that looks like a month-of-the-year factor, provided that it is also adjusted for momentum and residual volatility. By comparison, a very risk averse investor seeks refuge in the market factor. While Brandt, Santa-Clara, and Valkanov (2009) show that with a single characteristic an optimized portfolio resembles a characteristic-based long-short portfolio, when there are multiple characteristics the resulting portfolio is not spanned by the univariate characteristic-based factors.

\section{Portfolio Selection}

\subsection{Algorithm}

In Brandt, Santa-Clara, and Valkanov's (2009) algorithm, the vector $\theta$ is estimated to maximize a concave loss function over $T$ periods:

$$
\max _{\theta} \sum_{t=0}^{T-1} \frac{\left(1+r_{p, t+1}\right)^{1-\gamma^{*}}}{1-\gamma^{*}}\left(\frac{1}{T}\right)
$$

by allowing portfolio weights to depend on observable stock characteristics:

$$
r_{p, t+1}=\sum_{i=1}^{N_{t}}\left(\bar{\omega}_{i, t}+\frac{1}{N_{t}} \theta^{\prime} x_{i, t}\right) \cdot r_{i, t+1},
$$

where: $x_{i, t}$ is the $K$-vector of zero-mean characteristics on firm $i$, measurable at time $t$; $\bar{\omega}_{i, t}$ is the weight of stock $i$ in the (value-weighted) market portfolio at time $t$; and $N_{t}$ is the number of stocks in the sample at time $t \unlhd^{3}$ Unlike Brandt, Santa-Clara, and Valkanov or DeMiguel, Martín-Utrera, Nogales, and Uppal (2020), we do not identify $\gamma^{*}$, the parameter used to generate a feasible portfolio strategy in (1) with the relevant statistical loss function. Instead, we consider a statistical loss function (alternatively "an investor") that takes the same form as (1) indexed by $\gamma$ (the curvature of the statistical loss function which is pre-determined and fixed). From this perspective, $\gamma^{*}$ is a choice variable-a tool to manage estimation risk.

Our empirical design fixes $\gamma$, the curvature of the statistical loss function. Our interest is in out-of-sample statistical comparisons across portfolios generated by various feasible portfolio rules-from the perspective of this nonlinear statistical loss function. An eligible portfolio rule consists of a subset of characteristics, the in-sample period construction protocol, and the curvature of the in-sample loss function, $\gamma^{*}$. By definition, the optimal in-sample portfolio is obtained using all characteristics and by setting $\gamma^{*}$ equal to $\gamma$. Whether this is also true out of sample is an empirical question. If there is a positive relationship between an optimal portfolio's insample even-order moments and its noise (i.e., higher out-of-sample even-order moments) then

\footnotetext{
${ }^{3}$ We condition only on information that is available to investors at the time the portfolios are formed. This avoids the overconditioning bias analyzed by Boguth, Carlson, Fisher, and Simutin (2011).
} 
using a more concave loss function than the investor's utility function (i.e., $\gamma^{*}>\gamma$ ), may generate portfolios that are preferred to those obtained by constraining $\gamma^{*}$ to equal $\gamma$.

\subsection{Data and specifications}

Our sample spans January 1955 through December 2018. Our out-of-sample period comprises the 44 years 1975 - 2018. We use the following characteristics: momentum (M), the book-tomarket ratio $(\mathrm{V}), \log$ size $(\mathrm{S})$, beta $(\beta)$, market model residual standard deviation $\left(\sigma_{\epsilon}\right)$, last year's same-month return, $\left(r_{t-12}\right)$, and the average same-month return over the preceding five years, $(\bar{r})$. Momentum is the stock's compounded return from month $t-13$ though $t-2$. Equity market capitalization is the market value of the company's outstanding shares (aggregated across all share classes) at time $t-2$. Book value is obtained from the Compustat database for the most recent fiscal year-end between $t-6$ and $t-18$. Letting $B$ be one plus the ratio of book value to equity market capitalization, the book-to-market ratio is the natural $\log$ of $B$. Size is equity market capitalization. We estimate beta and the residual standard deviation by regressing monthly returns from months $t-60$ through $t-1$ on the CRSP value-weighted index. Thus the stocks in the January 1960 sample have no missing data from January 1955 through December 19594

We obtain all characteristic and return data from the merged CRSP-Compustat file on WRDS. To be eligible for inclusion in the sample in month $t$, a stock must have no missing returns in the CRSP database for the previous 60 months (in order to construct the characteristics in month $t$ ), and it must have a positive book value in the Compustat database for a fiscal year-end between $t-6$ and $t-18$. We obtain the US Consumer Price Index from the Federal Reserve (FRED) $)^{5}$ and use this to construct a minimum size criterion of $\$ 50$ million in January 1990 dollars. Stocks whose market capitalization is less than this inflation-adjusted size criterion are excluded from the sample. This excludes stocks with market capitalization less than $\$ 11.5$ million in January 1960, and $\$ 99.1$ million in December 2018. We next exclude the smallest $10 \%$ of stocks that meet all inclusion criteria prior to January 1978, when the first Nasdaq stocks enter the sample, and the smallest $20 \%$ afterwards ${ }^{6}$ If the stock return is missing in month $t$, we look to the CRSP delisting return. If that is missing, we substitute $-30 \%$ for NYSE- and AMEX-listed stocks and $-50 \%$ for Nasdaq stocks.

There are 389 (exclusively New York Stock Exchange) stocks in the final sample in January

\footnotetext{
${ }^{4}$ Our focus on out-of-sample statistics militates a long time-series. Starting in 1955 constrains our choice of characteristics. We use characteristics that can be directly computed from market prices, and the book value from the CRSP-Compustat merged file.

${ }^{5}$ We extracted the series CPIAUCSL: consumer price index for all urban consumers: all items.

${ }^{6}$ Green, Hand, and Zhang (2017) and Hou, Xue, and Zhang (2020) highlight the importance of removing microcap stocks from studies of return predictability.
} 
1960. The sample size jumps in August, 1967, from 674 to 875 when stocks listed on the American Stock Exchange are eligible for inclusion in our sample. The largest jump in sample size is in January 1978 (from 1,000 to 1,420 stocks) when Nasdaq stocks are eligible to enter our sample. The maximum number of stocks is 2,291 in April, 2006. There are 1,848 stocks in our sample in August 2008 and 1,617 stocks on the last date in our sample, December 2018.

We normalize and standardize the characteristics so that the mean is zero and the standard deviation is one. Inspection of (2) shows that optimal portfolio weights will thereby sum to unity for any value of $\theta$. This also means that the characteristics are observationally equivalent to shrinkage values. For example, let $\beta$ be a stock's OLS beta. Consider a prior-weighted beta, such as $\beta^{S}=.5 \cdot \beta+.5 \cdot 1$. The normalized $\beta^{S}$ are identical to the normalized $\beta$. This implicit shrinkage mitigates the usual concerns about outliers in characteristic space so we do not winsorize the characteristics. A single observation $\left(\Psi_{i, t}\right)$ comprises stock $i$ 's return in month $t, r_{i, t}$, as well as the vector of characteristics, measurable at month $t-1$, for stock $i, i=1, \ldots, N_{t}$. Importantly, $\Psi_{i, t}$ also includes the market capitalization of stock $i$ at $t-1$, since the passive portfolio at $t$ (i.e., the portfolio when the $\theta$-vector is zero) is the market-weighted portfolio at $t-1$.

Because financial firms' leverage is potentially different from that of non-financial firms we use two alternative treatments of the book-to-market ratio 7 In the first, we treat the book-to-market ratio the same as other characteristics: we standardize it using all stocks, and allow it to tilt their weights away from value-weighting. In the second treatment we exclude financial firms from this characteristic's standardization, and set their standardized book-to-market ratio to zero so that their weights are invariant with respect to this characteristic. This alternative treatment means that there are two versions of every characteristic set that includes the book-to-market ratio. We refer to the book-to-market ratio under the treatment that exempts financial firms as $V_{-f}$.

Excluding the alternative treatment of the book-to-market ratio, there are 127 possible sets of our 7 characteristic variables. We consider 48 characteristic sets, including each of the six characteristics as a singleton, and all seven variables together (last year's same-month return is only used in conjunction with the five-year average same-month return). 23 of these 48 include the book-to-market ratio, and each of these sets has two alternative treatments, which means that we use 71 unique sets of characteristics/treatments. The base 48 sets, maximized over the book-to-market treatment are reported in Table 1 . We consider 14 values of $\gamma^{*}: 2, \ldots, 13,16,22$.

The first in-sample period comprises the 180 months of January 1960 through December 1974. We use this sample to solve for the $\theta$-vector which we use to construct the out-of-sample feasible portfolio returns over the next 12 months (January through December, 1975). We estimate a new

\footnotetext{
${ }^{7}$ This issue is discussed in Barber and Lyon (1997). The proportion of financial stocks in our sample changes dramatically over the period from $1.5 \%$ in January 1960 to $11.9 \%$ in January 1978, to $21.6 \%$ in July 1994 , and ending at $19.9 \%$ in December 2018.
} 
$\theta$-vector at the beginning of each out-of-sample year. The out-of-sample period consists of the 528 months from January 1975 through December 2018. We obtain the $\theta$-vector using all available data through the end of year $T-1$ for $T=1, \cdots, 44$ under the updating protocol. Under the rolling protocol we use only the previous 180 months at the end of each year (so in Year 1, the two protocols produce the same $\theta$-vector). Since the portfolio is defined by $\theta$, there is no restriction on the available set of stocks in each of the out-of-sample months. The last out-of-sample year is 2018, so the last optimization/ $\theta$ estimation (applied to the 12 months of 2018, out of sample) uses 696 months under the updating protocol, and the 180 months from 2003 through 2017 under the rolling protocol. This means that we optimize expected utility (and estimate the $\theta$-vector) from each of the 1,988 cases (71 characteristic/treatment sets $\times 14$ values of $\gamma^{*} \times 2$ protocols) 44 times-at the end of each in-sample year.

\subsection{Empirical design}

The possibility that investors are learning about the data generating process, the fact that optimal portfolio selection amplifies estimation error, and the possibility of intertemporal changes in the conditional return distribution argue for analyzing optimal portfolios on an out-of-sample basis. We use a resampled-out-of-sample procedure to construct sampling distributions of the functions of interest, such as certainty equivalent, portfolio loading on factors, portfolio skewness, etc. The data in month $t$ consists of the $N_{t}$-row vector $\Psi_{t}$, with elements $\Psi_{i, t}$, for $i=1, \ldots, N_{t}$. We stack these $T$ row-vectors (of varying lengths) to form the array $\boldsymbol{\Psi}$. This is not a traditional panel since stock $i$ at time $t$ is different from stock $i$ at any other time, and as noted, the number of columns is different for each row.

Our resampling is nonparametric cross-sectional bootstrap (see Kapetanios 2008), motivated by the single period optimization problem with out-of-sample inference. It is nonparametric since we draw from the raw data. It assumes that returns are temporally independent conditional on $\boldsymbol{\Psi}$. As such, $\boldsymbol{\Psi}_{\boldsymbol{t}}^{*}$ is a cross sectional resample from $\boldsymbol{\Psi}_{\boldsymbol{t}}$, for months $t=1, \ldots, T$. Consistency holds under $N$-asymptotics (as the number of stocks in each period increases), since we can view $\theta$ as a GMM estimator. Preserving the original time series in all resamples allows us to evaluate the effects of structural instability on out-of-sample portfolios, and also maintains the cyclical (month of the year) and serial dependence patterns inherent in the design of $\boldsymbol{\Psi}_{\boldsymbol{t}}$. A resampled draw for month $t$ resamples (with replacement) an $N_{t}$-row vector from $\boldsymbol{\Psi}_{\boldsymbol{t}}$. Thus each pseudosample consists of the same number of observations in each period as the original sample, we preserve the calendar structure of the original data, and we maintain the same in-sample estimation, outof-sample evaluation periods as in the data. We also maintain the temporal structure of the investment opportunity set. In the original data $\boldsymbol{\Psi}_{\boldsymbol{t}}$ includes the raw values of the characteristics 
as well as the market weight of the stock at $\boldsymbol{t} \mathbf{- 1}$. Once we draw a resampled cross-section from $\boldsymbol{\Psi}_{\boldsymbol{t}}$ we normalize (to 0-mean characteristics), standardize (to characteristics with unit variances), and construct the value-weighted market weights for each resampled stock. Thus our resampling design preserves the integrity of the investment opportunity set at each $t$. This critically includes the relationship between stocks' characteristics and sizes. We also maintain the integrity of the seasonality in the data. The month of January, for example occurs only once in any 12-month cycle, and its timing is deterministic. The matrix of characteristics at $t$ is not independent of the size of each stock at $t$. We manage concerns about unmodeled temporal dependencies by maintaining the out-of-sample test design with each bootstrapped sample.

We also stress that we are not relying on resampling to evaluate the algorithm. We use out-ofsample validation. In each of our pseudosamples time plays the same role as it does in generating the data. We use resampling to construct sampling distributions of the out-of-sample properties, such as certainty equivalent and market exposure, of the optimal portfolio rules.

Once we have a pseudosample draw we obtain a $\theta$-vector by maximizing (1) at the end of year 15 , for a given feasible portfolio rule. We use this $\theta$-vector to construct the out-of-sample portfolio returns in each of the next 12 months. We repeat the optimization and out-of-sample portfolio return construction at the end of each year $16, \ldots, 59$. Thus we obtain $44 \theta$-vectors and 528 monthly feasible portfolio returns from each pseudosample. We take 10,000 pseudosamples to construct the sampling distributions for each feasible portfolio rule.

We use the empirical Monte Carlo small sample distributions of the certainty equivalent return to select the optimal feasible portfolio rule. As econometricians we view each of the pseudosamples as being equally likely. We consider that Portfolio A statistically significantly dominates Portfolio $\mathrm{B}$ if the $2.5 \%$ ile value of the sampling distribution of A's certainty equivalent return (the lower bound of the $95 \%$ statistical confidence interval) is greater than the $97.5 \%$ ile value of the sampling distribution of B's certainty equivalent return. Therefore we rank portfolio rules using the $2.5 \%$ ile value of the out-of-sample certainty equivalent return's sampling distribution. So the portfolio that we identify as optimal for a given loss function, indexed by $\gamma$ is maximally dominant, and not dominated by any other portfolio. However, as stressed by Lewellen, Nagel, and Shanken (2010 p. 185) the sampling error in stock returns is large relative to mean returns so we report confidence intervals of all of our functions of interest in order "to make sampling issues more transparent."

\section{Algorithm efficacy: Noise and estimation risk}

Table 1 shows the sampling distribution of the out-of-sample certainty equivalent returns on the optimal portfolios under each of the three loss functions, conditional on each of the 48 characteristic sets, maximized over the dimensions of $\gamma^{*}$, sample construction (rolling or updating), and 
book-to-market treatment (when relevant). The global optimal portfolios (i.e., maximally dominant over all feasible portfolio rules) are emboldened. The higher of the two benchmarks-equallyweighted and value-weighted portfolios of sample stocks-for each investor are also provided for comparison. This table shows that restricting $\gamma^{*}$ to equal $\gamma$ leads to poor out-of-sample performance, that this effect and the optimal characteristic set vary across $\gamma$, and that the updating sample construction protocol is preferred to rolling.

Panel A contains results for the $\gamma 2$ loss function, where the global optimal characteristic set consists of momentum, log size, beta, average same-month return, and residual standard deviation 8 The globally optimal portfolio is achieved with $\gamma^{*}=3$, updating sample construction, and characteristic set: momentum, log size, beta, average same-month return and residual volatility. This portfolio's mean (95\% confidence interval) certainty equivalent (basis points per month) $302(257,352)$, dominating the equally-weighted benchmark with its $95 \%$ ile certainty equivalent confidence interval of $(101,105)$. Seven of the 48 characteristic sets generate optimal portfolios that are not statistically dominated by the global optimal. For all of these seven, the optimal $\gamma^{*}$ is higher than 3; only one (that drops beta from the optimal set) has four characteristics. The others all have five, six, or seven characteristic variables. Fixing the characteristic set for this loss function, the rolling protocol is only preferred to updating when the book-to-market ratio is included in the characteristic set. But there are characteristic sets that include the book-to-market ratio where updating outperforms rolling.

Panel B shows the optimal portfolios from the perspective of the $\gamma 5$ loss function. In this case the global optimal portfolio is achieved with characteristics: momentum, log size, average same-month return, and residual standard deviation; $\gamma^{*}=10$; and updating sample construction. The mean (95\% confidence interval) certainty equivalent (basis points per month) on this portfolio is $152(133,170)$. This portfolio dominates this loss function's equally-weighted benchmark with its $95 \%$ ile certainty equivalent confidence interval of $(57,62)$. For this loss function there are nine alternative characteristic sets that generate portfolios that are not statistically dominated by the global optimal portfolio. Only one of these sets has three characteristics, all others have four or more. As was the case for the less concave loss function in Panel A, rolling sample construction is only preferred to updating when the characteristic set includes the book-to-market ratio.

Panel $\mathrm{C}$ reports the results from the perspective of the power utility function with $\gamma=9$. Here the global optimal portfolio is achieved with $\gamma^{*}=16$; characteristic set: momentum, $\log$ size, and residual standard deviation; and the updating sample construction. The global optimal portfolio's mean (95\% confidence interval) certainty equivalent (basis points per month) is 62

\footnotetext{
${ }^{8}$ As the only statistical loss functions that we consider are power utility functions, we will write $\gamma k$ loss function to mean "power utility function with coefficient of relative risk aversion, $\gamma=k$." We will use the phrases $\gamma k$ loss function and $\gamma k$ investor interchangeably.
} 
$(44,77)$, which dominates the value-weighted benchmark with its $95 \%$ ile certainty equivalent of $(4,15)$. For this loss function 21 of the 48 optimized portfolios' $95 \%$ certainty equivalent confidence intervals include zero; 14 of the 48 portfolios dominate the benchmark and are not statistically dominated by the global optimal portfolio. These include two which condition on only a single characteristic: either beta or residual volatility.

For each loss function, Table 1 Panel D isolates the characteristic set containing the bookto-market ratio which produces the highest $2.5 \%$ ile certainty equivalent return for each of the three loss functions. This panel shows the effects of treating financial firms differently as well as sample construction. In all three cases the choice of sample construction is more important than the treatment of financial stocks. And for these characteristic sets, updating always outperforms rolling for all three loss functions and for either treatment of financial firms.

Table 2 shows the effect of varying the curvature of the in-sample loss function $\gamma^{*}$ and the in-sample construction protocol on out-of-sample portfolio certainty equivalent returns for the globally optimal characteristic set and another characteristic set for each of the three loss functions. This table shows the importance of using $\gamma^{*}>\gamma$ as a tool to mitigate estimation risk. It also shows that once $\gamma^{*}$ reaches a critical level above the loss function's curvature of $\gamma$, further increases in $\gamma^{*}$ have fairly small effects on the resulting portfolio's certainty equivalent returns.

Table 2 Panel A shows the effects of changing $\gamma^{*}$ from $2, \ldots, 12$ for the $\gamma 2$ loss function and the optimal characteristic set along with the characteristic set that includes all characteristics except momentum. This case provides an example of the well-documented problem of the poor outof-sample properties of in-sample optimal portfolios. For the globally optimal characteristic set restricting $\gamma^{*}$ to equal $\gamma$ creates a feasible portfolio rule whose mean certainty equivalent is $-42 \%$, with $95 \%$ confidence interval, $(-100 \%, 3 \%)$ per month. $9^{9}$ Increasing $\gamma^{*}$ holding all else constant shows that much of the estimation risk in this context is the result of overfitting. Increasing the cost of even moments-expressed in terms of odd moments-has a dramatic salutary effect. Fixing $\gamma^{*}$ there are 142 optimal portfolios (across the characteristic sets and sample formation protocol). Restricting $\gamma^{*}=\gamma=2$ causes 94 of these 142 portfolios' $2.5 \%$ ile out-of-sample certainty equivalent return to be $-100 \%$.

Overfitting is more severe when the book-to-market ratio replaces momentum in the characteristic set. For this characteristic set, with either updating or rolling, $\gamma^{*}$ must exceed 5 in order for the $2.5 \%$ ile of the certainty equivalent's sampling distribution to exceed $-100 \%$. The maximally dominant portfolio with this characteristic set is obtained with $\gamma^{*}=8$ under the updating protocol and $\gamma^{*}=9$ under the rolling protocol. Both of these portfolios are statistically

\footnotetext{
${ }^{9}$ The certainty equivalent in a single draw is $-100 \%$ when at least one of the 528 monthly returns in the out-of-sample period is less than $-100 \%$.
} 
significantly dominated by the global optimal portfolio for the loss function indexed with $\gamma=2$.

Table 2 Panel B shows the out-of-sample certainty equivalent returns of optimal portfolios from the perspective of the $\gamma 5$ loss function. Under the updating protocol this loss function is less sensitive to estimation risk than is the $\gamma 2$ loss function. Further, estimation risk is more severe under the rolling protocol. Table 1 showed that this investors' optimal characteristic set consists of momentum, log size, average same-month return, and residual volatility. The updating protocol is optimal, and the optimal value of $\gamma^{*}$ is 10 . As is the case with the less concave loss function, the optimal value of $\gamma^{*}$ varies with the characteristic set and protocol. In general, a higher value of $\gamma^{*}$ is needed to mitigate overfitting under the rolling protocol than under the updating protocol. Here restricting $\gamma^{*}=\gamma$ results in a $2.5 \%$ ile certainty equivalent return of $-100 \%$ in 18 of the 71 cases with the updating protocol, and 83 of the 142 cases (under both protocols) out-of-sample certainty equivalent 95\%ile confidence intervals include zero. Eight of these 71 portfolios statistically dominate the benchmark, and 10 are not statistically dominated by the global optimal portfolio.

As in Table 1, Table 2 Panel $\mathrm{C}$ shows that the optimal portfolio for the power utility loss function with $\gamma=9$ is achieved with feasible portfolio rule comprising the updating protocol, $\gamma^{*}=16$, and characteristic set: momentum, log size, and residual volatility. The $2.5 \%$ ile of the out-of-sample certainty equivalent's sampling distribution is less than zero in 112 of the 142 optimal in-sample portfolios generated when $\gamma^{*}=\gamma=9$. Imposing this constraint produces eight portfolios that statistically dominate the value-weighted index. Whereas, Table 1 Panel C shows that 15 (of the 48) characteristic sets produce portfolios that dominate this benchmark under the updating protocol with $\gamma^{*}$ unconstrained.

In general, for all three loss functions overfitting is more severe under the rolling protocol than the updating protocol. One manifestation of this is that the optimal value of $\gamma^{*}$ under the former is greater than under the latter. For example for the $\gamma 2$ loss function the best rolling portfolio is achieved using all seven characteristics and $\gamma^{*}=6$. For the $\gamma 5$ investor the optimal rolling portfolio is achieved with $\gamma^{*}=13$. Panel $\mathrm{C}$ shows that when the characteristic set includes average same-month return, there is no value of $\gamma^{*}$ that produces a positive $2.5 \%$ ile certainty equivalent for the power utility function with $\gamma=9$ under the rolling protocol. This is not the case with the updating protocol.

Table 2 also shows that the effects of overfitting are not monotonic in $\gamma$. These effects are more pronounced for the $\gamma 2$ investor and the $\gamma 9$ investor than for the $\gamma 5$ investor. In fact, under the updating sample formation, using characteristics: momentum, size, beta, average samemonth return, and residual volatility, restricting $\gamma^{*}=5=\gamma$ produces a portfolio that statistically dominates this investor's preferred market benchmark. 
All panels in both Tables 1 and 2 highlight the fact that the sampling variations of the certainty equivalent returns are large. The global optimal portfolio does not dominate any of the other portfolios in Table 2, for both the $\gamma 5$ loss function (Panel B) and the $\gamma 9$ loss function (Panel C). By contrast, the global optimal portfolio for the $\gamma 2$ loss function (Panel A) statistically dominates 29 of the other 43 portfolios in this panel. These large sampling variances highlight the usefulness of using the $2.5 \%$ ile of the (out-of-sample) certainty equivalent returns' sampling distribution to rank the portfolio rules.

These three loss functions are representative of others. The optimal characteristic set for power utility loss functions with $\gamma=3$ and $\gamma=4$ is the same as for the $\gamma 2$ loss function. The $\gamma 3$ loss function is maximized with $\gamma^{*}=5$, and the $\gamma 4$ loss function is maximized with $\gamma^{*}=7$. Similarly, the $\gamma 6$ and $\gamma 7$ loss functions have the same optimal characteristic set as the $\gamma 5$ loss function. The global out-of-sample maximum for both of these loss functions is achieved with $\gamma^{*}=12$, under the updating protocol. Both the $\gamma 8$ and $\gamma 10$ loss functions' global optimal portfolios are formed using the same characteristic set as the $\gamma 9$ loss function, and $\gamma^{*}$ of 16 . All loss functions prefer updating to rolling. The out-of-sample certainty equivalent return's $95 \%$ confidence interval of the optimal portfolio for the power utility function with $\gamma=10$ is $(22.5,62.9)$ basis points per month. An investor with these preferences would choose to invest in Treasury bills over any equity position.

As noted in Section 2.2, we have Monte Carlo out-of-sample results for 1,988 portfolios. The 48 optimal portfolios in Table 1 have the highest $2.5 \%$ ile certainty equivalent across the dimensions of $\gamma^{*}$, protocol (rolling or updating), and treatment of the book-to-market ratio. Since we consider 14 integer values of $\gamma^{*}$, each of these portfolios is the optimal of 56 portfolios for the 23 characteristic sets that include the book-to-market ratio, or 28 for the 25 characteristic sets that do not. Even though we do not estimate conditional moments, overfitting degrades the out-of-sample performance of the optimal portfolios. This is especially severe for the leastand most- concave loss functions that we consider. Using a loss function in-sample that places a higher penalty on extreme losses than the actual (out-of-sample) loss function mitigates the unwelcome effects of noise on portfolio weights.

\section{How characteristics affect optimal portfolios}

In this section we evaluate properties of the global optimal portfolio returns, and compare these to the benchmarks. We also isolate the effect of each characteristic on the sampling distributions of various portfolio properties. To this end, we keep $\gamma^{*}$ and the protocols fixed at their optimal values for each of the three loss functions indexed by $\gamma$, and vary the characteristic set. We start with the optimal set for each loss function, and remove each characteristic in turn. 
We examine the effect of each characteristic on certainty equivalent returns and unconditional portfolio moments in Section 4.1. We assess the complementarities between the characteristics by examining the effects of removing an optimal characteristic on the $\theta$ coefficients that link the remaining characteristics to portfolio weights in Section 4.2. In Section 4.3 we examine the role of each characteristic in increasing or decreasing portfolio exposure to the Fama-French-Carhart factors. We conclude this section by summarizing across these dimensions at the characteristic level.

\subsection{Certainty equivalents and unconditional moments}

Table 3 contains sampling distribution properties of: certainty equivalent returns, unconditional portfolio moments, and other portfolio properties for benchmarks and optimal portfolios from the perspectives of each of the three loss functions. Certainty equivalent returns are reported in basis points per month. The mean return, median return, return standard deviation, and interquartile return range are all reported in percentage (per month) terms. The robust measures of skewness $\left(S_{4}\right)$ and kurtosis $\left(K_{3}\right)$ are recommended by Kim and White (2003):

$$
S_{4}=\frac{\mu-r_{.5}}{\sigma}
$$

and

$$
K_{3}=\frac{\bar{r}_{.95}^{+}-\bar{r}_{.05}^{-}}{\bar{r}_{.5}^{+}-\bar{r}_{.5}^{-}}-2.63
$$

where: $\mu$ is the mean portfolio return over the 528-month out-of-sample period, $\sigma$ is the return standard deviation, $\bar{r}_{.95}^{+}$is the mean of the highest $5 \%$ of returns, $\bar{r}_{.05}^{-}$is the mean of the smallest $5 \%$ of returns, $\bar{r}_{.5}^{+}$is the mean of the top half of returns, and $\bar{r}_{.5}^{-}$is the mean of the bottom half of returns; and $r_{.5}$ is the median out-of-sample return. 10

Panel A shows that the $\gamma 2$ loss function strictly prefers the equally-weighted portfolio to the value-weighted portfolio of sample stocks. The $\gamma 5$ loss function is indifferent between the two statistically. An investor whose utility function matches the $\gamma 9$ loss function would prefer the value-weighted portfolio, but is so averse to risk that she would prefer the risk-free asset (offering an average of 37 basis points per month) to the value-weighted portfolio, whose $95 \%$ ile certainty equivalent return confidence interval is only $(4,15)$ basis points per month. Both benchmarks are more leptokurtic than the normal distribution, and both are significantly negatively skewed.

Panel B shows eight portfolios from the $\gamma 2$ loss function's perspective, starting with this loss function's global optimal portfolio. This portfolio has a $2.5 \%$ ile certainty equivalent of 258 basis

\footnotetext{
${ }^{10}$ As per Kim and White (2003), in our pseudosamples $S_{4}$, the Pearson skewness coefficient is similar to, and more reliable than $S_{3}$, the Bowley skewness coefficient-integrated over the tail size. Similarly $K_{3}$, the Hogg coefficient, is more reliable than $K_{4}$, the Crow and Siddiqui parameter.
} 
points per month-which statistically dominates the equally-weighted benchmark. Table 1 shows the optimal value of $\gamma^{*}$ is 3 in this case, so we fix $\gamma^{*}=3$ to evaluate the comparative statics relating to the characteristics of the other seven portfolios in this panel.

The 95\%ile confidence interval of the $\gamma 2$ loss function's global optimal portfolio's annualized Sharpe ratio is $(1.07,1.27)$, which is significantly larger than the equally-weighted portfolio's $(0.61,0.64)$. This portfolio's skewness and kurtosis are not significantly different from the benchmark, although unlike the benchmark, this portfolio is symmetric. This portfolio uses leverage to exploit the conditional cross-sectional return distribution, and to take on significantly more risk than the benchmark.

Dropping momentum from the optimal characteristic set increases the sampling standard deviation of the resulting portfolio rule's out-of-sample monthly certainty equivalent return from 106.1 to $3,366.8$ basis points, and the range of its $95 \%$ confidence interval from 94 to 10,279 basis points. Unlike the optimal portfolio, this portfolio does not dominate the benchmark, despite the fact that none of the moments or properties shown in Table 3 differ significantly from the global optimal portfolio. The (asymmetric) loss function amplifies the effect that removing momentum has on the sampling distribution of the minimum out-of-sample return. In $2.5 \%$ of the 10,000 pseudosamples the minimum optimum portfolio monthly return is less than $-115 \%$, and in half of the samples the minimum return is less than $-79 \%$. By contrast the $2.5 \%$ ile minimum return from the global optimal portfolio rule is $-68 \%$. This confirms the evidence from Table 2 that adding momentum to the other four optimal characteristics reduces overfitting. This complementary property of momentum can only be appreciated out of sample, and with a non-linear loss function. We will explore this aspect of momentum in more detail as we further analyze the nature of these portfolios in the next subsections.

Dropping log size from the optimal portfolio characteristic set significantly lowers the certainty equivalent return to $(170,248)$, the Sharpe ratio to $(0.83,1.03)$, and the portfolio median return from $(426,619)$ to $(253,402)$ basis points per month. Unlike the globally optimal portfolio, this portfolio is significantly more leptokurtic than the benchmark. It is also the only portfolio in this table that is positively skewed. Size is the only characteristic whose marginal addition to the others results in a significant increase in the median out-of-sample return. In contrast with momentum, removing log size from the characteristic set has virtually no effect on the resulting optimal portfolio rule's out-of-sample minimum monthly return.

On the margin, adding residual volatility to the other four optimal conditioning characteristics also significantly increases the certainty equivalent return. Adding this characteristic does not significantly alter any of the first four moments, but the Sharpe ratio is significantly increased from $(0.84,1.01)$. Removing either $\log$ size or residual volatility from the optimal characteristic 
set constrains this risk tolerant, $\gamma 2$ loss function's ability to take advantage of the conditional cross-section. By contrast, beta is not as important in constructing the conditional distribution. Removing beta from the optimal characteristic set does not significantly reduce certainty equivalent return, and (unlike the removal of momentum), does not generate a portfolio which fails to dominate the benchmark.

Adding average same-month return to the other four optimal characteristics significantly increases portfolio leverage from $(417,522 \%)$ to $(541,699 \%)$, while increasing the $2.5 \%$ ile of the minimum monthly return from $-86 \%$ to $-68 \%$. The marginal effects of average same-month return include a significantly higher out-of-sample portfolio expected return and Sharpe ratio. It has the largest marginal effect on reducing kurtosis, although this effect is not statistically significant.

Adding the book-to-market ratio to the global optimal characteristic set lowers the $2.5 \%$ ile certainty equivalent return from 258 to 247 basis points per month, but has no statistically significant effects on the unconditional moments or portfolio leverage ${ }^{11}$ The certainty equivalent effect is due to the slightly higher kurtosis and lower minimum return. This suggests that the book-to-market ratio is redundant in the context of the five optimal characteristics.

Substituting the book-to-market ratio for log size in the context of the other four optimal characteristics extenuates in-sample overfitting, as the width of the out-of-sample certainty equivalent return's $95 \%$ confidence interval increases from 94 to 127 basis points. This effect is the result of an increase in the sampling variance of the minimum return, whose $2.5 \%$ ile drops to $-86 \%$ with this characteristic set.

Table 3 Panel C provides basic unconditional out-of-sample properties of 8 portfolios from the perspective of the $\gamma 5$ loss function. As Table 1 shows, this loss function's optimal (outof-sample) portfolio is formed using the characteristic quartet: momentum, log size, average same-month return, and residual volatility; $\gamma^{*}=10$, and the updating protocol. Accordingly, all of the portfolios in Panel B are generated using $\gamma^{*}=10$. Here the optimal portfolio has an out-of-sample certainty equivalent return of $(133,170)$ basis points per month and Sharpe ratio of $(1.15,1.32)$, which are more than twice those of the benchmark. This portfolio is much less levered than the $\gamma 2$ loss function's optimal portfolio, with an average investment of $244 \%$ of equity. This portfolio's mean, median, standard deviation, and kurtosis are all significantly higher than the benchmark's. However, its monthly return interquartile range $(557,695)$ basis points per month is not significantly higher than the benchmark's $(590,619)$. Interquartile range is a robust measure of scale that is not distorted by the distributions' leptokurtosis.

\footnotetext{
${ }^{11}$ In this context there is virtually no difference between the protocol that exempts financial stocks from conditioning on the book-to-market characteristic and the protocol that does not differentiate financial stocks in this way. The $95 \%$ ile certainty equivalent confidence interval for the former case is $(246.2,353.4)$, using $\gamma^{*}=3$. This is consistent with the results shown in Table 2 Panel D for this loss function. In that case we optimize over $\gamma^{*}$ as well. For this set of characteristics the optimal $\gamma^{*}=4$. Here we use $\gamma^{*}=3$ for the purpose of comparative statics.
} 
For the $\gamma 5$ loss function, removing either momentum or average same-month return does not significantly lower the certainty equivalent return. However, because conditioning on momentumin conjunction with the other characteristics-reduces overfitting, the portfolio formed without momentum does not significantly dominate the benchmark. Dropping momentum increases the width of the certainty equivalent return's $95 \%$ confidence interval from 38 to 90 basis points per month. Removing average same month return lowers leverage significantly from $(123 \%, 168 \%)$ to $(96 \%, 121 \%)$, but otherwise has no significant effects on any of the moments or properties reported in Table 3 Panel $\mathrm{C}$, for the $\gamma 5$ loss function.

Removing log size from the optimal characteristic set for the $\gamma 5$ loss function results in a significant drop in certainty equivalent return-which is due to a significant drop in the first moment, and not the second moment. This is also reflected in the resulting portfolio's significantly lower Sharpe ratio $(0.87,1.03)$. This suggests that log size is particularly useful in the context of momentum and residual volatility at boosting expected return. The residual standard deviation is the only characteristic for the $\gamma 5$ loss function whose removal not only significantly lowers the certainty equivalent return (to $(58,73)$ basis points per month), but also results in a portfolio that does not dominate the benchmarks. We will return to the role of residual volatility in this case in Section 4.3

Adding the book-to-market ratio to the four optimal characteristics for the $\gamma 5$ loss function does not improve the portfolio in any way. Adding this characteristic has little effect on the resulting portfolio's out-of-sample properties. Relative to the optimal for this loss function the minimum monthly return is lowered, manifesting in a 10 basis point per month reduction in the out-of-sample certainty equivalent return's $2.5 \%$ ile sampling value. Similarly, adding beta to the optimal characteristic set does nothing to improve the portfolio, and has almost no discernible effect on any of the out-of-sample properties shown in Panel C.

The $\gamma 9$ loss function's optimal portfolio, and related portfolios selected using $\gamma^{*}=16$ are reported in Table 3 Panel D. While this loss function prefers the riskless asset to the valueweighted portfolio of sample stocks, cross-sectional predictability is reliable enough to lever 1.77 times, conditioning portfolio weights on momentum, log size, and residual volatility. This loss function's optimal portfolio return standard deviation of $(433,477)$ basis points per month is not statistically different from the value-weighted portfolio's $(427,437)$, while its expected return $(164,189)$ and median return $(170,208)$ are significantly higher than this benchmark's $(102,110)$ and $(121,144)$, respectively. Figure 1 shows the densities of these two portfolios, which are easy to compare visually because of their similar scales. The figure and the summary statistics show that the optimal portfolio is significantly more leptokurtic than the benchmark, and has a significantly lower interquartile range $(4.33,5.02)$ than the benchmark $(5.04,5.42)$. Together these features 
suggest that the portfolio is so different from the Gaussian distribution that the standard deviation is not a sufficient statistic for the scale. That is, the optimal portfolio's departures from normality imply that the standard deviation is not a proper measure of the portfolio's riskiness from the perspective of the $\gamma 9$ power utility function.

Figure 1 and Table 3 also show that unlike the benchmark, the optimal portfolio is not negatively skewed. The area in the figure between $-10 \%$ and $0 \%$ (monthly return) is clearly higher under the benchmark, whereas the opposite is true between $2.5 \%$ and $10 \%$. The difference is especially large between $-6 \%$ and $-8 \%$. The $\gamma 9$ loss function's heightened asymmetry precludes the portfolio from attaining the Sharpe ratio $(0.99,1.14)$ of the more risk tolerant loss functions (the $\gamma 5$ optimal portfolio's Sharpe ratio is $(1.15,1.32)$ ).

For this loss function and optimal characteristic set removing momentum does not mitigate overfitting, as was the case with the flatter loss functions and higher dimensional optimal characteristic spaces. Although dropping momentum does result in a lower $2.5 \%$ ile minimum monthly return-despite the reduced dimensionality of the parameter space and significantly lower leverage. The portfolio mean, median, and standard deviation are all significantly lower with momentum removed from the optimal set, and $\gamma^{*}=16$.

Removing log size from the optimal characteristic triplet for this loss function does not significantly lower the certainty equivalent return, but leverage and the Sharpe ratio are significantly lowered. As was the case for the $\gamma 5$ loss function, removing residual volatility significantly adversely affects every desirable attribute of the optimal portfolio. In fact, for this loss function the optimal portfolio conditional only on momentum and size is dominated by the riskless asset.

As with the other two loss functions, adding the book-to-market ratio to the optimal characteristic set adds only estimation risk, so that it is redundant in this context. A utility function with $\gamma=9$ is too risk-averse to benefit from the boost to portfolio expected return provided by the average same-month return. Adding beta and average same-month return to the optimal characteristic triplet, using $\gamma^{*}=16$ amplifies the role of in-sample noise. The width of the certainty equivalent $95 \%$ confidence interval increases from 33 to 77 basis points, and the resulting portfolio does not dominate the value-weighted benchmark or the risk-free rate.

\subsection{Coefficients linking portfolio weights to characteristics}

Empirical designs with linear loss functions evaluate a characteristic's efficacy by the statistical significance of the coefficient on that characteristic (e.g., DeMiguel, Martín-Utrera, Nogales, and Uppal 2020; and Freyberger, Neuhierl, and Weber 2020). This is not the case with our empirical design. We are interested in the size and sign of the coefficients from alternative specifications in order to understand how characteristics affect portfolio properties and how they complement, 
or substitute for, one another. However, the statistical significance of a $\theta$ coefficient is neither a necessary nor sufficient condition for a characteristic's efficacy. This is because we select the optimal model based on out-of-sample certainty equivalent, not in-sample properties of $\theta$. Table 4 reports the sampling distributions of the $\theta$ coefficients that link portfolio weights to characteristics for the 22 portfolios in Table 3. For each feasible portfolio rule there are 44 (annual) estimates of the $\theta$-vector. We average these across the 44 years; the table reports the small sample properties of these annual averages. All three globally optimal portfolios have significantly positive coefficients on momentum, and significantly negative coefficients on size and residual volatility. The sense in which momentum complements the other characteristics is evident in the comparative statics in this table. For all three loss functions removing momentum does not significantly alter any of the $\theta$ coefficients on the remaining characteristics. This is consistent with the conclusion from the portfolio comparisons that the complementarity between momentum and the other optimal characteristics arises primarily out of sample.

With or without momentum, the algorithm puts more weight into stocks that are relatively small, have relatively low residual volatility, and relatively high average same-month average return based on the relationships between these variables and future returns (in the sample). Table 3 shows that when momentum is not part of the characteristic set these weights result in a portfolio with such degraded out-of-sample properties that it is unacceptable to the $\gamma 2$ loss function using $\gamma^{*}=3$. However, if a stock with higher weighting based on these four characteristics also has relatively low momentum, then adding momentum to the characteristic set lowers the weight- mitigating the effect of overfitting on portfolio formation. Similarly without momentum in the characteristic set, the algorithm will heavily short relatively large, high volatility stocks with low average same-month returns. Allowing the algorithm to complement these weights by also weighting momentum dramatically reduces the adverse effects of noise on the portfolio's performance (out of sample). Supplementing the predictive content of size, volatility, and samemonth return with momentum increases these characteristics' signal-to-noise ratio, resulting in improved out-of-sample performance. This property of momentum is especially highlighted by the asymmetric loss function.

In contrast to momentum, average same-month return is largely independent of the other characteristics-both in- and out of-sample. For both the $\gamma 2$ and $\gamma 5$ loss functions removing average same-month return from the respective optimal characteristic set does not induce a significant change in any of the other $\theta$ coefficients. Similarly, adding beta and average same-month return to the three optimal characteristics for the $\gamma 9$ loss function, with $\gamma^{*}=16$ (Panel $\mathrm{C}$ ) has little effect on the other three coefficients.

Beta is in the optimal characteristic set for only the least concave (most risk tolerant) loss 
function. Removing beta from this optimal characteristic set has no significant effects on the remaining $\theta$ coefficients. When $\log$ size is removed, the coefficient on beta becomes strictly positive, suggesting that high beta stocks with low residual volatility substitute for small stocks (also with low residual volatility). Table 3 shows that this substitution is less than satisfactory for the $\gamma 2$ loss function, as the certainty equivalent of the resulting portfolio is significantly less than the optimal portfolio.

When residual volatility is removed from the optimal characteristic set for the $\gamma 2$ loss function the $\theta$ coefficient on beta becomes strictly negative. There is something about high residual volatility stocks that all investors want to avoid, and about low residual volatility stocks that all risk averse investors want heightened exposure to. When this characteristic is not available investors use beta as an imperfect proxy. Table 3, Panel B shows that beta is a poor proxy because when it is the only volatility characteristic both the $\gamma 2$ loss function certainty equivalent return and Sharpe ratio are significantly lower than the optimal portfolio's (which also conditions on residual volatility). Furthermore, whereas removing residual volatility changes the sign of the $\theta$ coefficient on beta, removing beta does not significantly affect the $\theta$ coefficient on residual volatility (nor does it significantly change the certainty equivalent return).

Panel B of Table 4 reports comparative statics on the $\theta$ coefficients for optimal portfolios obtained with $\gamma^{*}=10$, starting with the global optimal portfolio from the perspective of the $\gamma 5$ loss function. In this case the $\theta$ coefficient on log size is significantly negative, with $95 \%$ sampling confidence interval $(-5.2,-2.8)$. When we remove residual volatility from the optimal characteristic set this coefficient becomes insignificant, $(-0.1,0.8)$. Small stocks, per se, are not attractive to this loss function. Small stocks with low residual volatility are however.

Since the global optimal portfolio for the loss function indexed with $\gamma=9$ uses three characteristics, Table 4 Panel $\mathrm{C}$ shows sampling distribution properties of the $\theta$ coefficients on these characteristics and related comparative statics. When residual volatility is removed from the triplet the sign of the coefficient on size switches significantly from negative $(-3.4,-1.4)$ to positive $(0.1,1.2)$. And the coefficient on momentum switches from significantly positive $(0.7,2.0)$ to insignificantly different from $0,(-0.1,1.4)$.

Table 4 Panel D reports the sampling standard deviations and correlations of the $\theta$ estimators for the three optimal portfolios. These reflect in-sample complementarities and substitutabilities. The largest correlations in absolute value are the positive 0.92 and 0.91 correlations between the coefficients on log size and residual volatility in the optimal portfolios for the $\gamma 5$ and $\gamma 9$ loss functions, respectively. This is consistent with the comparative statics results that show that for these loss functions, small stocks are only appealing if they have low residual volatility. This effect is dependent on the loss function, as the correlation between the coefficients on log size 
and residual volatility is negative $(-0.15)$ in the case of the optimal portfolio for the $\gamma 2$ loss function. The substitution effect between beta and residual volatility for the $\gamma 2$ loss function is seen in-sample in the -0.85 correlation between the $\theta$ estimators in this case. The in-sample correlation between size and momentum is negative for all three loss functions and increasing in absolute value in risk aversion. The same is true for the correlation between the $\theta$ coefficients on momentum and residual volatility.

All four $\theta$ coefficients on the globally optimal characteristics shared by the $\gamma 2$ and $\gamma 5$ loss functions drop significantly in absolute value as risk aversion increases. This is consistent with the hypothesis that conditioning on these characteristics shifts portfolio exposure to priced factors. This issue is explored further in the next subsection where we evaluate the effects of characteristics on factor exposures.

Comparing Tables 3 and 4 shows the tension between in-sample statistical significance of a characteristic's $\theta$ coefficient and that characteristic's efficacy in the out-of-sample loss function. Beta is included in the optimal characteristic set for the $\gamma 2$ loss function. However the $95 \%$ confidence interval on this $\theta$ coefficient is $(-1.6,7.5)$. Table 3 , Panel B shows that adding the book-to-market ratio to the optimal characteristic set for this loss functions lowers the $2.5 \%$ ile certainty equivalent by 10 basis points per month. However the $95 \%$ confidence interval on this characteristic's $\theta$ coefficient is $(1.2,6.8)$.

\subsection{Relationships between optimal portfolios and Fama-French-Carhart factors}

Table 5 Panel A decomposes the two benchmark and 22 characteristic-based portfolio returns (from Tables 3 and 4) using a linear projection of the 528 out-of-sample monthly excess returns on the four Fama-French-Carhart factors 12 The table reports the intercept from this regression ( $\alpha$ in basis points per month), along with the coefficients on each of the factors. The projection decomposes the variance into 11 components with the orthogonal component being the ratio of the residual variance to the total portfolio variance. The table only provides covariance terms whose sampling means exceed $5 \%$ of total variance, so the tabulated component means do not sum to unity. Panel B contains the correlation / standard deviation matrix of the four factors over the sample period.

The optimal portfolio for the $\gamma 2$ loss function largely lies outside the span of the four factors. Its mean alpha $(95 \%$ confidence interval) is $269(201,348)$ basis points per month, and $(61,75)$ percent of the (excess) return variance is not spanned by the factors. The portfolio gets less than

\footnotetext{
${ }^{12}$ These four factors are: the excess return on the CRSP value-weighted market index (MKT), the size factor (SMB), value factor (HML), and momentum factor (MOM). We obtain these along with the riskfree rate from Professor Kenneth French's website at Dartmouth. We use the 528 monthly returns in our out-of-sample period: January 1975 through December 2018.
} 
$10 \%$ of its variance from each of the market and size factors. This portfolio has significantly more exposure to the value $(12 \%, 21 \%)$ and momentum factors $(14 \%, 28 \%)$ than the benchmarks.

Dropping momentum from the characteristic set for this loss function changes the momentum factor loading from $(1.2,1.8)$ to $(-0.5,-0.1)$. The momentum factor's contribution to the portfolio variance falls from $(14 \%, 28 \%)$ to $(0 \%, 3 \%)$. Removing the characteristic momentum does not shift exposures into the other three Fama-French-Carhart factors. The percentage of variance that is orthogonal to these factors increases significantly from $(61 \%, 75 \%)$ to $(76 \%, 86 \%)$. This portfolio has the highest alpha of all the tabulated portfolios. Yet, Table 3 shows that the mean (95\% confidence interval) of this portfolio's out-of-sample certainty equivalent return is $-11 \%$ $(-100 \%, 2.8 \%)$ per month. This portfolio with the highest alpha does not statistically dominate the benchmark. This is a graphic example of the importance of the loss function in assessing the predictive content of characteristics.

Section 4.1 and Table 3 show that adding log size to the other optimal characteristics for the $\gamma 2$ loss function significantly increases the portfolio's certainty equivalent return, expected return, and Sharpe ratio. Table 5 shows that this result is not the result of a significant increase in the portfolio's alpha. Instead, it is due to the effects that conditioning on size have on the portfolio's optimal SMB exposure. When log size is not in the conditioning set the portfolio's outof-sample loading on SMB is significantly negative $(-1.5,-0.9)$. This is primarily the result of the negative coefficient on residual volatility for this characteristic set (as shown in Table 4 Panel A). Adding size to the characteristic set shifts the portfolio's SMB loading to significantly positive $(0.6,1.4))$. Similarly, when portfolio weights are not size-dependent, HML exposure is statistically insignificant $(-0.1,0.4)$, and significantly lower than that of the equally-weighted benchmark. Adding size to the characteristic set raises the HML exposure to $(1.6,2.4)$ ). Furthermore, adding log size to the other characteristics significantly decreases the portfolio's exposure to the market factor. This loss function uses log size and residual volatility to create a portfolio that is largely market-neutral.

Replacing the coefficient log size with the book-to-market ratio shows that size is uniquely suited to zeroing-out the market. Table 5 shows that the optimal portfolio obtained using the updating protocol, $\gamma^{*}=3$, and characteristic set: momentum, book-to-market ratio, beta, average same-month return, and residual volatility has the same HML exposure as the globally optimal portfolio, however it inherits one-fourth of its out-of-sample variance from the market-significantly more than the global optimal portfolio's 3\%. Furthermore, substituting the book-to-market ratio for size significantly decreases the SMB loading from $(0.6,1.4)$ to $(-1.0,-0.3)$.

Consistent with the implications of Tables 3 and 4, removing beta from the optimal characteristic set for the $\gamma 2$ loss function has little effect on the relationships between the factors and 
the out-of-sample returns of the resulting portfolio.. Similarly, adding beta to the optimal characteristic set for the $\gamma 5$ loss function has little effect on the resulting portfolio's factor regression and variance decompositions.

Removing average same-month return significantly lowers the optimal portfolio's alpha-for optimal portfolios from both the $\gamma 2$ and $\gamma 5$ loss functions. For the $\gamma 2$ loss function the resulting portfolio has significantly higher exposure to the momentum factor than the globally optimal portfolio. HML becomes significantly more important for the $\gamma 5$ loss function. Nevertheless, $(45,51)$ percent of the variance of the $\gamma 5$ loss function's optimal portfolio conditioned on the characteristic triplet momentum, log size, and residual volatility (using $\gamma^{*}=10$ ), is not explained by the Fama-French-Carhart factors. This characteristic triplet is also the optimal set for the $\gamma$ 9 loss function (using $\gamma^{*}=16$ ). Holding this set of characteristics fixed, increasing $\gamma^{*}$ from 10 to 16 shifts the portfolio's exposure away from momentum to the market. The momentum factor's loading decreases from $(0.51,0.68)$ to $(0.36,0.48)$ with a corresponding decrease in variance coverage from $(22,32)$ percent to $(12,21)$ percent. Movement into the market is reflected by the increase in factor loading from $(0.45,0.56)$ to $(0.54,0.63)$ and variance coverage from $(13,23)$ percent to $(26,40)$ percent.

Panel B of Table 1 and Panel C of Table 3 show that the $\gamma 5$ loss function can use residual volatility as a singleton to outperform the benchmark. Table 4, Panel B shows that the $\theta$ average (95\% confidence interval) coefficient on residual volatility in this case is $-2.3(-3.1,-1.5)$. Table 3 , Panel $\mathrm{C}$ shows that this results in a portfolio whose out-of-sample expected return is significantly lower than the benchmark. Since this portfolio has a significantly higher certainty equivalent than the benchmark, it follows that its standard deviation and interquartile range are also significantly lower than the benchmark. It is also significantly more leptokurtic than the benchmark. Table 5 provides evidence about how this risk reduction in accomplished. This portfolio has a mean (95\% confidence interval) market beta of $0.76(0.73,0.79)$, and derives $43 \%(46 \%, 49 \%)$ of its variance from the market. It has a significant negative loading on SMB, and derives roughly one-fourth of its variance from this factor. The portfolio has a mean loading of 0.4 on HML, and derives $10 \%$ of its variance from this factor. Table 5 Panel B shows that the market and SML are positively correlated, the opposite signed loadings means that the cross effect between these two factor reduces this portfolios variance attributed to the two factors in isolation by $22 \%$. Similarly the negative correlation between the market and HML coupled with loadings of the same sign reduce the net variance contribution from these two factors by $13 \%$. This means that $21 \%$ of the portfolio's variance lies outside the span of the FFC factors, and the portfolio has an alpha of $29.9(22.4,37.4)$ basis points per month.

These results are curious since as we have seen above (in Section 4.1), removing residual 
volatility from the optimal characteristic set for the $\gamma 5$ loss function, significantly lowers the expected portfolio return from $2.4(2.2,2.7)$, per cent per month to $1.4(1.4,1.5)$ per cent per month, with little change in the portfolio returns' interquartile range. Table 4, Panel C shows the in-sample complementarities for residual volatility with $\gamma^{*}=10$. Removing residual volatility significantly increases the $\theta$ coefficient on $\log$ size from $-4.0(-5.2,-2.8)$ to $0.4(-0.2,0.8)$. The loading on MOM, the momentum factor, drops significantly from $0.6(0.5,0.7)$ to $0.3(0.2,0.4)$. The loading on HML drops significantly from $1.1(1.0,1.2)$ to $-0.15(-0.2,-0.1)$. As discussed above, the FFC factors account for less than $50 \%$ of the variance of the optimal portfolio, with the market contributing $19 \%(13 \%, 25 \%)$. However, restricting the coefficient on residual volatility to 0 results in a portfolio for which the market accounts for $87 \%(83 \%, 91 \%)$ of its variance. These complementarities show that it is difficult to isolate residual volatility's effects, as it is closely linked to the size and value factors.

Table 4 shows that the $\theta$ coefficient linking $\log$ size to portfolio weights is significantly negative $(-3.4,-1.4)$ in the optimal portfolio rule for the $\gamma 9$ investor. This tilts the portfolio toward small stocks (and away from large-cap stocks). Yet Table 5 shows that this portfolio has a significantly negative SMB loading $(-0.3,-0.1)$. This follows from the fact that conditioning on residual volatility tilts the portfolio toward large-cap stocks. Table 5 shows that when this loss function conditions only on residual volatility and momentum, the loading on SMB is significantly more negative than for the global optimal portfolio, $(-0.7,-0.6)$. This loss function uses log size to offset the excess exposure to large-cap stocks that results from conditioning on residual volatility. This significantly increases the portfolio's expected return from $(1.2,1.4)$ to $(1.6,1.9)$, per cent per month; alpha, from $(16,31)$ to $(40,62)$ basis points per month; and Sharpe ratio, from $(0.8,0.9)$ to $(1.0,1.1)$.

Beta substitutes for log size as a means of increasing the loading on SMB when the latter characteristic is removed from the optimal characteristic set for the $\gamma 2$ loss function. Optimally the coefficient on beta is statistically insignificantly different from 0 (Table 4, Panel A). But when size is removed from the optimal characteristic set, this becomes significantly positive $(2.9,8.8)$. The portfolio dramatically increases its exposure to the market portfolio, in order to offset the large firm tilt that results from the negative coefficient on residual volatility. And despite the tilt toward high beta stocks still has a significant negative loading on SMB $(-1.5,-0.9)$. The efficacy of the characteristic log size stems from its complementarity with residual volatility-a phenomenon that is manifest for all three loss functions we highlight.

Consider the use of leverage by the most risk-averse loss function. Despite preferring the riskless asset to the market, this loss function's optimal portfolio uses $77 \%$ leverage to exploit predictable properties in the cross-section. Green and Hollifield (1992) propose an ingenious 
argument as to why well diversified portfolios may have extreme short positions. They point out that if there is a single factor on which all stocks load positively (e.g., in the CAPM or the pricing kernel), then minimizing portfolio variance might be accomplished by finding a diversified portfolio with a zero loading on this factor. The $\gamma 9$ loss function provides some insight as to whether this technique to reduce factor exposure appeals to highly risk-averse investors. This portfolio has significantly more exposure to the market and HML factors than the $\gamma 2$ loss function's optimal portfolio. The portfolio does use short positions to reduce exposure to the market factor. However, the optimal portfolio's expected return, 182, $(169,196)$ and median return, 196, $(176,217)$ (basis points per month) are significantly higher than those of the market: 108, (103, 112) and 135, $(123,147)$, respectively. This most risk-averse loss function finds exposure to the value factor and momentum factors desirable. She uses characteristics to create portfolios with exposure to these factors, as well as to factors lying outside the span of the Fama-French-Carhart factors.

\subsection{Characteristic comparative statics: Summaries}

\subsubsection{Momentum}

All investors' global optimal portfolios have a significant positive coefficient on momentum. For the $\gamma 2$ loss function momentum serves to significantly reduce overfitting. Table 1, Panel A and Table 2, Panel A show that the value of $\gamma^{*}$ required to overcome overfitting is highest for the characteristic sets which do not include momentum. Table 3, Panel A shows that when momentum is removed from the optimal conditioning set in this case the $2.5 \%$ ile of the certainty equivalent sampling distribution drops from $2.6 \%$ per month to to -100this behavior is unique across the characteristics. Table 4 Panel A shows that removing momentum from the optimal characteristic set does not have a significant effect on the coefficients on the remaining characteristics, consistent with the premise that the salutary effects of momentum arise out-of-sample. This effect is also seen in Table 4, Panel D: the in-sample correlations between the coefficient on momentum and that on both residual standard deviation and average same-month return are close to zero. This beneficial effect of reducing overfitting decreases in $\gamma$, as the tilting toward, and dimensionality of the other characteristics diminishes. Nevertheless, conditioning on momentum is the only tool investors have to gain the desired exposure to the momentum factor which accounts for $15-20 \%$ of the return variance of all three loss functions' optimal portfolios.

\subsubsection{Log size}

All optimal portfolio rules have a significant negative $\theta$ coefficient on size. Adding size to the set of other optimal characteristics significantly increases the Sharpe ratio in all three cases. Certainty equivalent increases significantly for power utility investors indexed by both $\gamma=2$ and $\gamma=5$. Expected return and alpha increase significantly for the $\gamma 5$ and $\gamma 9$ loss functions. This 
effect is muted for the $\gamma 2$ loss function which substitutes high beta for small stocks. For the more risk-averse loss functions adding size increases the SMB and HML loadings and alpha, while also shifting the variance outside the span of the FFC factors. Adding size to the other characteristics increases the $\theta$ coefficient on residual volatility. The SMB loading absent size is significantly negative, so adding size significantly reduces the effect of SMB on the portfolio. Without size in the characteristic set, the portfolios of the $\gamma 5$ and $\gamma 9$ loss functions derive $25 \%$ of their variance from SMB. Adding size shrinks this to less than 4\%. For the most risk averse investor we consider, Table 4, Panel $\mathrm{C}$ shows that the $\theta$ coefficient on log size is significantly negative, whereas Table 5, Panel A shows that this factor's loading on SMB is significantly negative. This arises because this investor wants a large stock tilt, but significantly less than the tilt on this factor induced by shifting toward low volatility stocks.

\subsubsection{Residual volatility}

All optimal portfolio rules have a significant negative $\theta$ coefficient on residual volatility. For the power utility investor with $\gamma=2$, adding this characteristic to momentum, log size, average samemonth return, and beta significantly increases resulting portfolio's out-of-sample Sharpe ratio and certainty equivalent return. This occurs with no significant changes in the portfolio's alpha or variance decomposition with respect to the Fama-French-Carhart factors. The complementary set of characteristics for the more risk averse investors, indexed with $\gamma=5$ and $\gamma=9$ does not include beta, so in both of these cases adding residual volatility significantly increases alpha and shifts the portfolio outside the span of the four factors. In these two cases adding residual volatility significantly increases the portfolio's exposure to the momentum and value factors. This demonstrates the complementarity between a measure of volatility and momentum and size. When the investor cannot down-weight stocks with high residual volatility she does not want exposure to the momentum factor. Exposure to momentum only increases out-of-sample expected utility when the investor can put less weight in stocks that had high momentum but that were also highly volatile. Table 5 shows that the complementarity with log size is an insample effect: adding residual volatility to the complementary set has a statistically significant on the coefficient that weights size. By contrast, as noted above, the complementarity with momentum is only observed out-of-sample, as adding residual volatility does not significantly affect the weighting coefficient on momentum. In this case it is conditioning on residual volatility that offsets the (in-sample) overfitting that harms the momentum investor.

\subsubsection{Average same-month return}

While average same-month return is the only characteristic that can be used as a singleton by our most risk-tolerant investor to outperform the benchmark its predictive content is too risky for our most risk-averse loss functions to exploit. Adding it to the other four characteristics for 
the $\gamma 2$ loss function has no effect on any of their $\theta$ coefficients, suggesting limited in-sample complementarity. The resulting portfolio is less leptokurtotic and has a higher minimum monthly return. While neither of these effects is statistically significant each is amplified by the loss function. This suggests that as is the case with momentum, conditioning on average same-month return mitigates some of the estimation risk inherent in the other characteristics. This manifests in the reduction in the width of the certainty equivalent's 95\% confidence interval from 116 to 94 basis points, which when scaled by the mean certainty equivalent is a drop from 63 to $32 \%$.

\subsubsection{Beta}

Beta only enters the optimal characteristic set for our most risk-tolerant loss functions, and has a minimal effect in the context of the other optimal characteristics. Its complementarity to log size and residual volatility are important in assessing their marginal contributions. Removing log size from the optimal characteristic changes the average $\theta$ coefficient on beta from insignificant to significantly positive, as the portfolio uses beta as an imperfect inverse proxy for size. Similarly, removing residual volatility from the optimal characteristic changes the average $\theta$ coefficient on beta to significantly negative. Downweighting (residual) volatility is important for all optimal portfolios. In the absence of residual volatility beta serves as an imperfect substitute 13

\subsubsection{Book-to-market ratio}

All of our optimal portfolios load significantly on the value factor (HML), without conditioning on the book-to-market ratio which has been historically associated with value. Although the bookto-market ratio has a positive, statistically significant $\theta$ coefficient when added to the optimal characteristic set for the $\gamma 2$ and $\gamma 9$ loss functions, its marginal effects on the resulting portfolios are very small, the $2.5 \%$ ile sampling value of the minimum monthly return is lowered, as is certainty equivalent's $2.5 \%$ ile sampling value. The desired positioning of the portfolio relative to the FFC factors is achieved by residual volatility and size, in combination. Conditional on that information the book-to-market ratio is redundant, and adding it to optimal characteristic set increases sampling variation, reflected in the width of out-of-sample certainty equivalent's 95\% confidence interval. This result supports Kozak, Nagel, and Santosh's (2020) premise that characteristics and risk factors do not span the same space.

\section{Conclusions}

An important goal of linking portfolio weights to characteristics, avoiding the intermediate step of estimating conditional moments, is mitigation of estimation risk, which is known to plague optimal portfolio selection. However, we show that the benefits from conditioning on observable characteristics are largely lost to overfitting when an agent obtains portfolio weights by max-

\footnotetext{
${ }^{13}$ This finding is consistent with Liu, Stambaugh, and Yuan (2018) who show that the negative relationship between beta and expected return is not robust to controlling for idiosyncratic volatility.
} 
imizing her own utility function on sample data. Regularizing by ratcheting up risk aversion for the purpose of in-sample utility maximization mitigates overfitting, and allows a risk averse investor to profit from the predictive information contained in the characteristic and return data. These out-of-sample utility gains derive from shifting into stocks that are jointly small, have low residual volatility, and positive momentum. The gains derive from complementarities across these characteristics, as no one characteristic can be used to produce utility gains by all loss functions.

The Fama-French-Carhart factors do not span the space of priced factors in the market. The most risk tolerant investor's optimal portfolio alpha is over 200 basis points per month. But this is not a free lunch. Increasing risk aversion shifts the portfolio toward the four factors-significantly decreasing alpha. Nevertheless, less than one-fourth of the variance of the optimal portfolio for a power utility investor with relative risk aversion of five is driven by the market. Furthermore more than half of this portfolio's return variance is unrelated to the four Fama-French-Carhart factors.

Whereas we have filtered our results through different loss functions, and considered "out-ofsample" returns, our empirical approach is not normative. Consistent with computer-intensive, machine-learning methods, we use the out-of-sample period to select the optimal feasible portfolio rule and evaluate performance. Because we are interested in comparing non-nested alternatives fraught with estimation risk this is a design for inference not prescription. This is also reflected in our empirical design which ignores trading costs and assumes away short-selling constraints (although in the presence of either static optimization is not appropriate).

How can these results be reconciled with equilibrium? Several of the assumptions underlying our design are likely violated. First, we consider only a static optimization problem. This is of course optimal if the investment opportunity set does not change over time. That seems unlikely. Second, we optimize only within the set of publicly traded equities. This can understate the appeal of the market depending on how it correlates with other forms of wealth (for example real estate and human capital). Another way to interpret this is that the market portfolio, or even a convex combination of the Fama-French-Carhart factors, is a poor proxy for the stochastic discount factor. 


\section{References}

Aït-Sahalia, Yacine and Michael W. Brandt, 2001, Variable selection for portfolio choice, Journal of Finance 56, 1297-1351.

Barber, Brad M. and John D. Lyon, 1997, Firm size, book-to-market ratio, and security returns: A holdout sample of financial firms, Journal of Finance 52, 875-883.

Barroso, Pedro and Pedro Santa-Clara, 2015, Momentum has its moments, Journal of Financial Economics 116, 111-120.

Best, Michael J. and Robert R. Grauer, 1991, On the sensitivity of mean-variance-efficient portfolios to changes in asset means: Some analytical and computational results, Review of Financial Studies $4,315-342$.

Boguth, Oliver, Murray Carlson, Adlai Fisher, and Mikhail Simutin, 2011, Conditional risk and performance evaluation: Volatility timing, overconditioning, and new estimates of momentum alphas, Journal of Financial Economics 102, 363-389.

Brandt, Michael W., Pedro Santa-Clara, and Rossen Valkanov, 2009, Parametric portfolio policies: Exploiting characteristics in the cross-section of equity returns, Review of Financial Studies 22, $3411-3447$.

Broadie, Mark, Mikhail Chernov, and Michael Johannes, 2009, Understanding index option returns, Review of Financial Studies 22, 4493-4529.

Cochrane, John H., 2011, Discount Rates, Journal of Finance 66, 1047-1108.

DeMiguel, Victor, Lorenzo Garlappi, and Raman Uppal, 2009, Optimal versus naive diversification: How inefficient is the 1/N portfolio strategy?, Review of Financial Studies 22, 1915-1953.

DeMiguel, Victor, Alberto Martín-Utrera, Francisco J. Nogales, and Raman Uppal, 2020, A transactioncost perspective on the multitude of firm characteristics, Review of Financial Studies 33, 21802222.

Feng, Guanhao, Stefano Giglio, and Dacheng Xiu, 2020, Taming the factor zoo: A test of new factors, Journal of Finance 75, 1327-1370.

Ferson, Wayne, 2013, Ruminations on investment performance measurement, European Financial Management 19, 4-13.

Freyberger, Joachim, Andreas Neuhierl, and Michael Weber, 2020, Dissecting characteristics nonparametrically, Review of Financial Studies 33, 2326-2377. 
Gennotte, Gerard, 1986, Optimal portfolio choice under incomplete information, Journal of Finance 41, 733-746.

Giglio, Stefano, Yuan Liao, and Dacheng Xiu, 2021, Thousands of alpha tests, The Review of Financial Studies 34, 3456-3496.

Goetzmann, William, Jonathan Ingersoll, Matthew Spiegel, and Ivo Welch, 2007, Portfolio performance manipulation and manipulation-proof measures, Review of Financial Studies 20, 15031546.

Green, Jeremiah, John R.M. Hand, and X. Frank Zhang, 2017, The characteristics that provide independent information about average U.S. monthly stock returns, Review of Financial Studies 30, 4389-4436.

Green, Richard C. and Burton Hollifield, 1992, When will mean-variance efficient portfolios be well diversified? Journal of Finance 47, 1785-1809.

Harvey, Campbell R., 2017, Presidential address: The scientific outlook in financial economics, Journal of Finance 72, 1399-1440.

Harvey, Campbell R., Yan Liu, and Heqing Zhu, 2016, ... and the cross-section of expected returns, Review of Financial Studies 29, 5-68.

Harvey, Campbell R. and Akhtar Siddique, 2000, Conditional skewness in asset pricing tests, Journal of Finance 55, 1263-1295.

Heston, Steve L. and Ronnie Sadka, 2008, Seasonality in the cross-section of stock returns, Journal of Financial Economics 87, 418-445.

Hou, Kewei, Chen Xue, and Lu Zhang, 2020, Replicating anomalies, Review of Financial Studies 33, 2019-2133.

Jagannathan, Ravi and Tongshu Ma, 2003, Risk reduction in large portfolios: Why imposing the wrong constraint helps, Journal of Finance 58, 1651-1683.

Kadan, Ohad and Fang Liu, 2014, Performance evaluation with high moments and disaster risk, Journal of Financial Economics 113, 131-155.

Kan, Raymond and Guofu Zhou, 2007, Optimal portfolio choice with parameter uncertainty, Journal of Financial and Quantitative Analysis 42, 621-656.

Kapetanios, George, 2008, A bootstrap procedure for panel data sets with many cross-sectional units, The Econometrics Journal 11, 377-395. 
Kim, Tae-Hwan and Halbert White, 2003, On more robust estimation of skewness and kurtosis: Simulation and application to the S\&P 500 Index, Working Paper, University of California, San Diego.

Kozak, Serhiy, Stefan Nagel, and Shrihari Santosh, 2018, Interpreting factor models, Journal of Finance $73,1183-1223$.

Kozak, Serhiy, Stefan Nagel, and Shrihari Santosh, 2020, Shrinking the cross section, Journal of Financial Economics 135, 271-292.

Leland, Hayne, 1999, Beyond mean-variance: Performance measurement in a nonsymmetrical world, Financial Analysts Journal 27-36.

Lewellen, Jonathan, Stefan Nagel, and Jay Shanken, 2010, A skeptical appraisal of asset pricing tests, Journal of Financial Economics 96, 175-194.

Linnainmaa, Juhani T. and Michael R. Roberts, 2018, The history of the cross-section of stock returns, Review of Financial Studies 31, 2606-2649.

Liu, Jianan, Robert F. Stambaugh, and Yu Yuan, 2018, Absolving beta of volatility's effects, Journal of Financial Economics 128, 1-15.

MacKinlay, A. Craig and Luboš Pástor, 2000, Asset pricing models: Implications for expected returns and portfolio selection, Review of Financial Studies 13, 883-916.

Martin, Ian and Stefan Nagel, 2020, Market efficiency in the age of big data, Working paper, University of Chicago.

McLean, R. David and Jeffrey Pontiff, 2016, Does academic research destroy stock return predictability? Journal of Finance 71, 5-31.

Pástor, Luboš, 2000, Portfolio selection and asset pricing models, Journal of Finance 60, 179-223.

Pástor, Luboš and Robert F. Stambaugh, 2000, Comparing asset pricing models: An investment perspective, Journal of Financial Economics 56, 335-381.

Patton, Andrew, 2004, On the out-of-sample importance of skewness and asymetric dependence for asset allocation, Journal of Financial Econometrics 2, 130-168. 


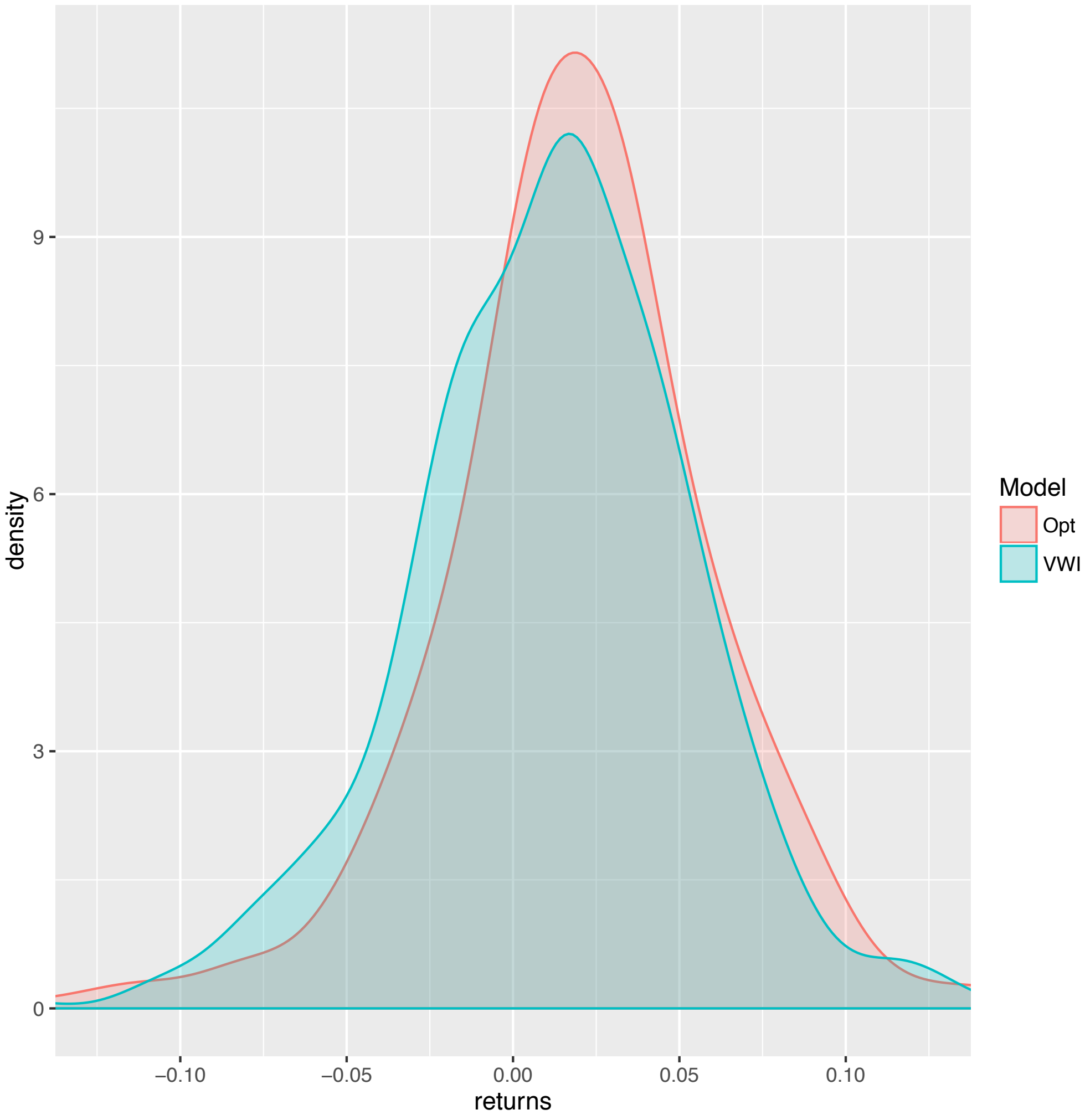

Figure 1. Return densities The monthly out-of-sample return densities of the valueweighted index (VWI) and global optimal portfolio for the power utility loss function with $\gamma=9$ obtained (using $\gamma^{*}=16$; allowing weights to depend on: momentum, $\log$ size, and residual standard deviation); and updating sample construction. Both densities are constructed using all returns from 10,000 pseudosamples of monthly returns from the 44-year out-of-sample period, 1975 - 2018. 


\section{Table 1}

Optimal $\gamma^{*}$ and protocol for each characteristic set:

Sampling properties of certainty equivalent returns

For each of the 48 characteristic sets, we report the value of $\gamma^{*}$ and protocol that generate the maximum 2.5\%ile certainty equivalent for each loss function, along with sampling statistics of the certainty equivalent. The characteristic symbols are: M: momentum, V: book-to-market ratio, S: $\log$ size, $\beta$ : from lagged 60 -month market model, $r_{t-12}$ : last-year's same-month return, $\bar{r}$ : average same-month return over the previous 5 years, $\sigma_{\epsilon}$ : standard deviation of lagged 60-month market model residual. $V_{-f}$ refers to the treatment in which financial stocks' weights are not affected by their book-to-market ratios. We report the marginal effects of this treatment in Panel D.

\section{Panel A: $\gamma 2$ loss function}

\begin{tabular}{|c|c|c|c|c|c|}
\hline \multirow[t]{2}{*}{$\begin{array}{l}\text { Characteristic } \\
\text { set / Benchmark }\end{array}$} & \multirow[t]{2}{*}{$\begin{array}{c}\text { Optimal } \\
\gamma^{*}\end{array}$} & \multirow[t]{2}{*}{ Protocol } & \multicolumn{3}{|c|}{$\begin{array}{c}\text { Resampled } \\
\text { Out of sample }\end{array}$} \\
\hline & & & $2.5 \%$ ile & Mean & $97.5 \%$ ile \\
\hline Equally-weighted portfolio & & & 100.6 & 102.7 & 104.8 \\
\hline $\mathrm{M}, \mathrm{V}, \mathrm{S}, \beta, r_{t-12}, \bar{r}, \sigma_{\epsilon}$ & 4 & Updating & 249.9 & 289.6 & 337.6 \\
\hline $\mathrm{M}, V_{-f}, \mathrm{~S}, \beta, r_{t-12}, \bar{r}$ & 6 & Rolling & 197.8 & 232.9 & 268.5 \\
\hline $\mathrm{M}, \mathrm{V}, \mathrm{S}, \beta, \bar{r}, \sigma_{\epsilon}$ & 4 & Updating & 250.7 & 291.2 & 335.0 \\
\hline $\mathrm{V}, \mathrm{S}, \beta, r_{t-12}, \bar{r}, \sigma_{\epsilon}$ & 8 & Updating & 164.2 & 192.0 & 218.9 \\
\hline $\mathrm{M}, V_{-f}, \mathrm{~S}, \beta, \sigma_{\epsilon}$ & 5 & Rolling & 184.3 & 212.2 & 242.1 \\
\hline $\mathrm{M}, V_{-f}, \mathrm{~S}, r_{t-12}, \bar{r}$ & 6 & Rolling & 187.8 & 221.6 & 256.5 \\
\hline $\mathrm{M}, \mathrm{V}, \mathrm{S}, \bar{r}, \sigma_{\epsilon}$ & 4 & Updating & 239.1 & 276.3 & 314.8 \\
\hline $\mathrm{V}, \mathrm{S}, \beta, r_{t-12}, \bar{r}$ & 9 & Rolling & 140.0 & 164.7 & 188.1 \\
\hline $\mathrm{V}, \mathrm{S}, \beta, \bar{r}, \sigma_{\epsilon}$ & 6 & Updating & 169.2 & 203.1 & 235.4 \\
\hline $\mathrm{M}, V_{-f}, \beta, \bar{r}, \sigma_{\epsilon}$ & 4 & Updating & 199.5 & 236.9 & 282.2 \\
\hline $\mathrm{M}, \mathrm{S}, \boldsymbol{\beta}, \overline{\boldsymbol{r}}, \boldsymbol{\sigma}_{\epsilon}$ & 3 & Updating & 257.4 & 301.6 & 351.7 \\
\hline $\mathrm{M}, \mathrm{V}, \mathrm{S}, \sigma_{\epsilon}$ & 5 & Updating & 181.8 & 201.0 & 221.1 \\
\hline $\mathrm{M}, V_{-f}, \mathrm{~S}, \beta$ & 5 & Rolling & 153.5 & 172.6 & 193.2 \\
\hline $\mathrm{V}, \mathrm{S}, \beta, \sigma_{\epsilon}$ & 5 & Updating & 115.0 & 131.3 & 149.5 \\
\hline$V_{-f}, \mathrm{~S}, r_{t-12}, \bar{r}$ & 7 & Rolling & 115.2 & 136.8 & 157.1 \\
\hline$\beta, r_{t-12}, \bar{r}, \sigma_{\epsilon}$ & 8 & Updating & 126.1 & 144.8 & 165.0 \\
\hline $\mathrm{M}, V_{-f}, \mathrm{~S}, \bar{r}$ & 4 & Updating & 178.3 & 207.8 & 238.0 \\
\hline $\mathrm{M}, \mathrm{S}, \beta, \bar{r}$ & 4 & Updating & 182.8 & 208.6 & 237.2 \\
\hline $\mathrm{M}, \mathrm{S}, \beta, \sigma_{\epsilon}$ & 5 & Updating & 182.5 & 205.6 & 231.1 \\
\hline $\mathrm{S}, \beta, \bar{r}, \sigma_{\epsilon}$ & 6 & Updating & 175.9 & 206.8 & 237.8 \\
\hline $\mathrm{M}, \mathrm{S}, \bar{r}, \sigma_{\epsilon}$ & 4 & Updating & 245.5 & 278.9 & 314.2 \\
\hline $\mathrm{M}, \beta, \bar{r}, \sigma_{\epsilon}$ & 4 & Updating & 178.4 & 210.5 & 246.6 \\
\hline $\mathrm{M}, V_{-f}, \bar{r}, \sigma_{\epsilon}$ & 5 & Updating & 181.4 & 212.5 & 244.2 \\
\hline $\mathrm{M}, r_{t-12}, \bar{r}$ & 5 & Updating & 122.7 & 137.2 & 153.3 \\
\hline $\mathrm{M}, V_{-f}, \mathrm{~S}$ & 4 & Rolling & 150.8 & 172.1 & 195.3 \\
\hline$V_{-f}, r_{t-12}, \bar{r}$ & 7 & Updating & 125.7 & 141.8 & 159.3 \\
\hline$r_{t-12}, \bar{r}, \sigma_{\epsilon}$ & 8 & Updating & 129.9 & 147.3 & 165.5 \\
\hline$V_{-f}, \mathrm{~S}, \sigma_{\epsilon}$ & 4 & Updating & 128.6 & 143.7 & 160.0 \\
\hline
\end{tabular}


Table 1 Continued

Optimal $\gamma^{*}$ and protocol for each characteristic set:

Sampling properties of certainty equivalent returns

\section{Panel A: $\gamma 2$ loss function}

\begin{tabular}{|c|c|c|c|c|c|}
\hline \multirow[t]{2}{*}{$\begin{array}{l}\text { Characteristic } \\
\text { set/Benchmark }\end{array}$} & \multirow[t]{2}{*}{$\begin{array}{l}\text { Optimal } \\
\gamma^{*}\end{array}$} & \multirow[t]{2}{*}{ Protocol } & \multicolumn{3}{|c|}{$\begin{array}{c}\text { Resampled } \\
\text { Out of sample }\end{array}$} \\
\hline & & & $2.5 \%$ ile & Mean & $97.5 \%$ ile \\
\hline $\mathrm{M}, \mathrm{S}, \sigma_{\epsilon}$ & 5 & Updating & 180.1 & 198.6 & 217.5 \\
\hline $\mathrm{M}, V_{-f}, \sigma_{\epsilon}$ & 5 & Rolling & 152.7 & 172.5 & 194.2 \\
\hline $\mathrm{S}, \bar{r}, \sigma_{\epsilon}$ & 6 & Updating & 184.4 & 215.1 & 244.1 \\
\hline $\mathrm{M}, \mathrm{S}, \bar{r}$ & 5 & Updating & 124.0 & 137.6 & 152.9 \\
\hline $\mathrm{M}, \bar{r}, \sigma_{\epsilon}$ & 4 & Updating & 158.9 & 182.9 & 210.2 \\
\hline $\mathrm{S}, \beta, \sigma_{\epsilon}$ & 4 & Updating & 122.9 & 138.9 & 156.2 \\
\hline$\beta, \sigma_{\epsilon}$ & 8 & Updating & 86.5 & 95.2 & 105.5 \\
\hline$V_{-f}, \sigma_{\epsilon}$ & 13 & Updating & 94.6 & 101.7 & 109.1 \\
\hline$V_{-f}, \mathrm{~S}$ & 5 & Updating & 84.9 & 92.9 & 101.0 \\
\hline $\mathrm{M}, \mathrm{S}$ & 5 & Updating & 100.9 & 108.9 & 117.4 \\
\hline $\mathrm{M}, \sigma_{\epsilon}$ & 6 & Updating & 116.9 & 127.1 & 138.0 \\
\hline $\mathrm{S}, \sigma_{\epsilon}$ & 3 & Updating & 134.9 & 149.9 & 165.4 \\
\hline $\mathrm{M}, \bar{r}$ & 5 & Updating & 122.9 & 137.2 & 152.7 \\
\hline M & 4 & Updating & 95.9 & 103.1 & 110.7 \\
\hline$V_{-f}$ & 7 & Updating & 89.7 & 96.3 & 103.3 \\
\hline S & 3 & Updating & 88.9 & 94.6 & 100.4 \\
\hline$\beta$ & 13 & Updating & 81.9 & 87.7 & 93.6 \\
\hline$r_{t-12}, \bar{r}$ & 5 & Updating & 117.3 & 131.1 & 146.5 \\
\hline $\bar{r}$ & 4 & Updating & 117.3 & 133.4 & 151.6 \\
\hline$\sigma_{\epsilon}$ & 11 & Updating & 94.7 & 101.6 & 108.5 \\
\hline
\end{tabular}

Panel B: $\gamma 5$ loss function

Equally-weighted portfolio

$\mathrm{M}, \mathrm{V}, \mathrm{S}, \beta, r_{t-12}, \bar{r}, \sigma_{\epsilon}$

$\mathrm{M}, V_{-f}, \mathrm{~S}, \beta, r_{t-12}, \bar{r}$

$\mathrm{M}, \mathrm{V}, \mathrm{S}, \beta, \bar{r}, \sigma_{\epsilon}$

$\mathrm{V}, \mathrm{S}, \beta, r_{t-12}, \bar{r}, \sigma_{\epsilon}$

$\mathrm{M}, V_{-f}, \mathrm{~S}, \beta, \sigma_{\epsilon}$

$\mathrm{M}, V_{-f}, \mathrm{~S}, r_{t-12}, \bar{r}$

$\mathrm{M}, \mathrm{V}, \mathrm{S}, \bar{r}, \sigma_{\epsilon}$

$\mathrm{V}, \mathrm{S}, \beta, r_{t-12}, \bar{r}$

$\mathrm{V}, \mathrm{S}, \beta, \bar{r}, \sigma_{\epsilon}$

$\mathrm{M}, V_{-f}, \beta, \bar{r}, \sigma_{\epsilon}$

$\mathrm{M}, \mathrm{S}, \beta, \bar{r}, \sigma_{\epsilon}$

$\mathrm{M}, V_{-f}, \mathrm{~S}, \sigma_{\epsilon}$

$\mathrm{M}, V_{-f}, \mathrm{~S}, \beta$

$\mathrm{V}, \mathrm{S}, \beta, \sigma_{\epsilon}$

$\mathrm{V}, \mathrm{S}, r_{t-12}, \bar{r}$

$\beta, r_{t-12}, \bar{r}, \sigma_{\epsilon}$

$\mathrm{M}, V_{-f}, \mathrm{~S}, \bar{r}$

\begin{tabular}{crrrr} 
& & 57.1 & 59.3 & 61.6 \\
11 & Updating & 115.6 & 142.4 & 165.0 \\
13 & Rolling & 94.7 & 129.2 & 152.7 \\
10 & Updating & 119.9 & 144.9 & 167.1 \\
22 & Updating & 72.4 & 98.8 & 118.0 \\
11 & Rolling & 107.4 & 122.1 & 137.3 \\
13 & Rolling & 78.0 & 110.6 & 133.0 \\
12 & Updating & 122.8 & 143.1 & 161.1 \\
22 & Updating & 64.4 & 86.1 & 102.2 \\
16 & Updating & 86.3 & 109.0 & 127.7 \\
13 & Updating & 86.1 & 110.1 & 130.2 \\
9 & Updating & 130.9 & 153.0 & 174.5 \\
11 & Updating & 111.8 & 124.0 & 136.2 \\
9 & Rolling & 98.4 & 110.6 & 123.1 \\
10 & Updating & 78.5 & 89.7 & 101.3 \\
16 & Updating & 39.2 & 55.7 & 68.3 \\
22 & Updating & 62.2 & 77.6 & 90.8 \\
10 & Updating & 79.6 & 95.6 & 112.5 \\
11 & Updating & 101.7 & 115.2 & 128.4 \\
\hline
\end{tabular}


Table 1 Continued

Optimal $\gamma^{*}$ and protocol for each characteristic set:

Sampling properties of certainty equivalent returns

\section{Panel B: $\gamma 5$ loss function}

\begin{tabular}{|c|c|c|c|c|c|}
\hline \multirow[t]{2}{*}{$\begin{array}{l}\text { Characteristic } \\
\text { set }\end{array}$} & \multirow[t]{2}{*}{$\begin{array}{c}\text { Optimal } \\
\gamma^{*}\end{array}$} & \multirow[t]{2}{*}{ Protocol } & \multicolumn{3}{|c|}{$\begin{array}{l}\text { Resampled } \\
\text { Out of sample }\end{array}$} \\
\hline & & & $2.5 \%$ ile & Mean & $97.5 \%$ ile \\
\hline $\mathrm{M}, \mathrm{S}, \beta, \sigma_{\epsilon}$ & 11 & Updating & 106.9 & 120.2 & 134.7 \\
\hline $\mathrm{S}, \beta, \bar{r}, \sigma_{\epsilon}$ & 16 & Updating & 85.3 & 108.5 & 126.3 \\
\hline $\mathrm{M}, \mathrm{S}, \overline{\boldsymbol{r}}, \sigma_{\epsilon}$ & 10 & Updating & 132.7 & 151.9 & 170.2 \\
\hline $\mathrm{M}, \beta, \bar{r}, \sigma_{\epsilon}$ & 9 & Updating & 95.5 & 113.2 & 131.1 \\
\hline $\mathrm{M}, V_{-f}, \bar{r}, \sigma_{\epsilon}$ & 16 & Updating & 88.0 & 107.0 & 123.5 \\
\hline $\mathrm{M}, r_{t-12}, \bar{r}$ & 11 & Updating & 62.5 & 69.5 & 76.8 \\
\hline $\mathrm{M}, V_{-f}, \mathrm{~S}$ & 8 & Rolling & 87.2 & 99.6 & 112.5 \\
\hline $\mathrm{V}, r_{t-12}, \bar{r}$ & 13 & Updating & 63.0 & 72.7 & 82.1 \\
\hline$r_{t-12}, \bar{r}, \sigma_{\epsilon}$ & 22 & Updating & 69.5 & 85.2 & 98.2 \\
\hline $\mathrm{V}, \mathrm{S}, \sigma_{\epsilon}$ & 9 & Updating & 89.2 & 100.1 & 111.0 \\
\hline $\mathrm{M}, \mathrm{S}, \sigma_{\epsilon}$ & 12 & Updating & 112.3 & 124.0 & 135.7 \\
\hline $\mathrm{M}, V_{-f}, \sigma_{\epsilon}$ & 11 & Rolling & 96.4 & 109.0 & 121.7 \\
\hline $\mathrm{S}, \bar{r}, \sigma_{\epsilon}$ & 16 & Updating & 93.6 & 117.3 & 135.7 \\
\hline $\mathrm{M}, \mathrm{S}, \bar{r}$ & 10 & Updating & 58.4 & 65.6 & 73.2 \\
\hline $\mathrm{M}, \bar{r}, \sigma_{\epsilon}$ & 11 & Updating & 92.9 & 107.2 & 121.0 \\
\hline $\mathrm{S}, \beta, \sigma_{\epsilon}$ & 9 & Updating & 81.2 & 91.3 & 101.5 \\
\hline$\beta, \sigma_{\epsilon}$ & 10 & Updating & 60.8 & 69.0 & 77.6 \\
\hline$V_{-f}, \sigma_{\epsilon}$ & 11 & Rolling & 60.5 & 69.2 & 77.7 \\
\hline$V_{-f}, \mathrm{~S}$ & 9 & Updating & 46.4 & 53.5 & 60.7 \\
\hline $\mathrm{M}, \mathrm{S}$ & 10 & Updating & 52.2 & 57.8 & 63.6 \\
\hline $\mathrm{M}, \sigma_{\epsilon}$ & 12 & Updating & 81.1 & 89.5 & 98.1 \\
\hline $\mathrm{S}, \sigma_{\epsilon}$ & 9 & Updating & 90.2 & 100.1 & 109.8 \\
\hline $\mathrm{M}, \bar{r}$ & 11 & Updating & 62.2 & 68.8 & 75.8 \\
\hline M & 10 & Updating & 54.6 & 59.8 & 65.2 \\
\hline$V_{-f}$ & 11 & Updating & 55.6 & 61.6 & 67.5 \\
\hline S & 6 & Updating & 50.7 & 55.2 & 59.6 \\
\hline$\beta$ & 7 & Updating & 58.9 & 65.4 & 71.8 \\
\hline$r_{t-12}, \bar{r}$ & 12 & Updating & 62.9 & 69.2 & 75.9 \\
\hline $\bar{r}$ & 11 & Updating & 62.5 & 69.5 & 76.8 \\
\hline$\sigma_{\epsilon}$ & 12 & Updating & 66.5 & 74.1 & 81.8 \\
\hline
\end{tabular}

Panel C: $\gamma 9$ loss function

Value-weighted portfolio

$\mathrm{M}, \mathrm{V}, \mathrm{S}, \beta, r_{t-12}, \bar{r}, \sigma_{\epsilon} \quad 22$

$\mathrm{M}, \mathrm{V}, \mathrm{S}, \beta, r_{t-12}, \bar{r} \quad 22$

$\mathrm{M}, \mathrm{V}, \mathrm{S}, \beta, \bar{r}, \sigma_{\epsilon} \quad 22$

$\mathrm{V}, \mathrm{S}, \beta, r_{t-12}, \bar{r}, \sigma_{\epsilon} \quad 22$

$\mathrm{M}, V_{-f}, \mathrm{~S}, \beta, \sigma_{\epsilon} \quad 16$

$\mathrm{M}, \mathrm{V}, \mathrm{S}, r_{t-12}, \bar{r}$

$\mathrm{M}, \mathrm{V}, \mathrm{S}, \bar{r}, \sigma_{\epsilon}$

$\mathrm{V}, \mathrm{S}, \beta, r_{t-12}, \bar{r}$

\begin{tabular}{lrrr} 
& 4.4 & 9.8 & 15.2 \\
Updating & -69.8 & 24.3 & 71.5 \\
Updating & -86.7 & 5.5 & 50.4 \\
Updating & -21.1 & 39.3 & 73.6 \\
Updating & -221.8 & -29.1 & 52.5 \\
Updating & 30.2 & 51.8 & 69.0 \\
Updating & -56.2 & 3.1 & 30.5 \\
Updating & -17.0 & 54.9 & 89.7 \\
Updating & -200.2 & -35.5 & 38.2 \\
\hline
\end{tabular}




\section{Table 1 Continued}

Optimal $\gamma^{*}$ and protocol for each characteristic set:

Sampling properties of certainty equivalent returns

\section{Panel C: $\gamma 9$ loss function}

\begin{tabular}{|c|c|c|c|c|c|}
\hline \multirow[t]{2}{*}{$\begin{array}{l}\text { Characteristic } \\
\text { set }\end{array}$} & \multirow[t]{2}{*}{$\begin{array}{r}\text { Optimal } \\
\gamma^{*}\end{array}$} & \multirow[t]{2}{*}{ Protocol } & \multicolumn{3}{|c|}{$\begin{array}{l}\text { Resampled } \\
\text { Out of sample }\end{array}$} \\
\hline & & & $2.5 \%$ ile & Mean & $97.5 \%$ ile \\
\hline $\mathrm{V}, \mathrm{S}, \beta, \bar{r}, \sigma_{\epsilon}$ & 22 & Updating & -62.6 & 23.2 & 65.6 \\
\hline $\mathrm{M}, V_{-f}, \beta, \bar{r}, \sigma_{\epsilon}$ & 22 & Updating & -72.6 & 5.9 & 46.9 \\
\hline $\mathrm{M}, \mathrm{S}, \beta, \bar{r}, \sigma_{\epsilon}$ & 16 & Updating & 13.1 & 61.3 & 89.7 \\
\hline $\mathrm{M}, V_{-f}, \mathrm{~S}, \sigma_{\epsilon}$ & 16 & Updating & 36.5 & 57.4 & 74.3 \\
\hline $\mathrm{M}, V_{-f}, \mathrm{~S}, \beta$ & 12 & Rolling & 32.8 & 52.4 & 68.0 \\
\hline $\mathrm{V}, \mathrm{S}, \beta, \sigma_{\epsilon}$ & 13 & Updating & 21.9 & 41.6 & 57.3 \\
\hline $\mathrm{V}, \mathrm{S}, r_{t-12}, \bar{r}$ & 22 & Updating & -148.3 & -49.9 & -4.7 \\
\hline$\beta, r_{t-12}, \bar{r}, \sigma_{\epsilon}$ & 22 & Updating & -99.4 & -7.8 & 38.3 \\
\hline $\mathrm{M}, V_{-f}, \mathrm{~S}, \bar{r}$ & 16 & Updating & -36.6 & 5.7 & 28.3 \\
\hline $\mathrm{M}, \mathrm{S}, \beta, \bar{r}$ & 16 & Updating & 13.0 & 46.2 & 67.1 \\
\hline $\mathrm{M}, \mathrm{S}, \beta, \sigma_{\epsilon}$ & 16 & Updating & 39.3 & 56.1 & 71.7 \\
\hline $\mathrm{S}, \beta, \bar{r}, \sigma_{\epsilon}$ & 22 & Updating & -68.8 & 21.2 & 64.9 \\
\hline $\mathrm{M}, \mathrm{S}, \bar{r}, \sigma_{\epsilon}$ & 16 & Updating & 19.2 & 68.6 & 95.9 \\
\hline $\mathrm{M}, \beta, \bar{r}, \sigma_{\epsilon}$ & 16 & Updating & 9.3 & 43.1 & 64.8 \\
\hline $\mathrm{M}, \mathrm{V}, \bar{r}, \sigma_{\epsilon}$ & 22 & Updating & -72.2 & 12.6 & 54.3 \\
\hline $\mathrm{M}, r_{t-12}, \bar{r}$ & 16 & Updating & -1.5 & 6.1 & 13.3 \\
\hline $\mathrm{M}, V_{-f}, \mathrm{~S}$ & 11 & Rolling & 19.0 & 35.6 & 49.0 \\
\hline $\mathrm{V}, r_{t-12}, \bar{r}$ & 16 & Updating & -25.2 & 1.1 & 15.8 \\
\hline$r_{t-12}, \bar{r}, \sigma_{\epsilon}$ & 22 & Updating & -94.2 & -0.8 & 44.8 \\
\hline $\mathrm{V}, \mathrm{S}, \sigma_{\epsilon}$ & 13 & Updating & 32.2 & 52.0 & 67.6 \\
\hline $\mathrm{M}, \mathrm{S}, \sigma_{\epsilon}$ & 16 & Updating & 44.2 & 62.2 & 77.4 \\
\hline $\mathrm{M}, V_{-f}, \sigma_{\epsilon}$ & 13 & Rolling & 21.6 & 47.4 & 66.1 \\
\hline $\mathrm{S}, \bar{r}, \sigma_{\epsilon}$ & 22 & Updating & -74.2 & 26.4 & 73.2 \\
\hline $\mathrm{M}, \mathrm{S}, \bar{r}$ & 22 & Updating & -8.5 & 0.4 & 8.9 \\
\hline $\mathrm{M}, \bar{r}, \sigma_{\epsilon}$ & 16 & Updating & 11.7 & 45.6 & 66.3 \\
\hline $\mathrm{S}, \beta, \sigma_{\epsilon}$ & 13 & Updating & 19.9 & 40.5 & 55.4 \\
\hline$\beta, \sigma_{\epsilon}$ & 12 & Updating & 11.5 & 27.8 & 40.8 \\
\hline $\mathrm{V}, \sigma_{\epsilon}$ & 11 & Rolling & 3.7 & 21.2 & 34.5 \\
\hline $\mathrm{V}, \mathrm{S}$ & 10 & Updating & -11.6 & -1.0 & 8.0 \\
\hline $\mathrm{M}, \mathrm{S}$ & 13 & Updating & -4.4 & 2.6 & 9.3 \\
\hline $\mathrm{M}, \sigma_{\epsilon}$ & 16 & Updating & 32.6 & 45.0 & 56.1 \\
\hline $\mathrm{S}, \sigma_{\epsilon}$ & 12 & Updating & 27.2 & 48.1 & 63.4 \\
\hline $\mathrm{M}, \bar{r}$ & 16 & Updating & -0.6 & 6.2 & 12.8 \\
\hline M & 13 & Updating & -1.9 & 4.4 & 10.5 \\
\hline$V_{-f}$ & 11 & Updating & 2.6 & 10.7 & 18.0 \\
\hline S & 10 & Updating & 1.4 & 7.2 & 12.8 \\
\hline$\beta$ & 6 & Updating & 21.5 & 31.2 & 39.9 \\
\hline$r_{t-12}, \bar{r}$ & 13 & Updating & 3.2 & 10.0 & 16.7 \\
\hline $\bar{r}$ & 16 & Updating & 6.9 & 12.8 & 18.6 \\
\hline$\sigma_{\epsilon}$ & 12 & Updating & 15.9 & 32.1 & 44.9 \\
\hline
\end{tabular}


Table 1 Continued

Optimal $\gamma^{*}$ and protocol for each characteristic set:

Sampling properties of certainty equivalent returns

Panel D: Alternative protocols for the book-to-market ratio (V)

Protocols Optimal Resampled

\begin{tabular}{lcc}
$\gamma^{*}$ & Out of sample \\
$2.5 \%$ ile & Mean & $97.5 \%$ ile \\
\hline
\end{tabular}

$\gamma 2$ loss function

Characteristic set: $\mathrm{M}, \mathrm{V}, \mathrm{S}, \beta, \bar{r}, \sigma_{\epsilon}$ :

Updating, $\mathrm{V} \quad 4 \quad 250.7$

Updating, $V_{-f} \quad 4 \quad 249.6$

$\begin{array}{lllll}\text { Rolling, V } & 7 & 205.3 & 245.1 & 286.2\end{array}$

\begin{tabular}{lllll} 
Rolling, $V_{-f}$ & 8 & 201.4 & 235.1 & 269.8 \\
\hline
\end{tabular}

\section{$\gamma 5$ loss function}

Characteristic set: $\mathrm{M}, \mathrm{V}, \mathrm{S}, \bar{r}, \sigma_{\epsilon}$ :

\begin{tabular}{llrll} 
Updating, V & 12 & 122.8 & 143.1 & 161.1 \\
Updating, $V_{-f}$ & 12 & 119.9 & 142.4 & 161.3 \\
Rolling, V & 16 & 76.4 & 122.3 & 148.7 \\
Rolling, $V_{-f}$ & 16 & 70.4 & 126.1 & 154.6 \\
\hline
\end{tabular}

\section{$\gamma 9$ loss function}

Characteristic set: $\mathrm{M}, \mathrm{V}, \mathrm{S}, \sigma_{\epsilon}$ :

\begin{tabular}{llrll} 
Updating, V & 16 & 33.0 & 55.5 & 72.5 \\
Updating, $V_{-f}$ & 16 & 36.5 & 57.4 & 74.3 \\
Rolling, V & 16 & 9.7 & 39.9 & 61.1 \\
Rolling, $V_{-f}$ & 13 & 25.8 & 53.5 & 73.1 \\
\hline
\end{tabular}




\section{Table 2}

\section{Estimation Risk: Sampling distribution of out-of-sample certainty equivalent returns (in basis points per month) -as portfolio rules vary}

Sampling properties of the out-of-sample certainty equivalent returns of various feasible portfolio rules. The utility function is isoelastic: $E(U)=\sum_{t=0}^{T-1} \frac{\left(1+r_{p, t+1}\right)^{1-\gamma}}{1-\gamma}\left(\frac{1}{T}\right)$. Panels A - C report results from the perspective of three different CRRA utility functions (or "investors") indexed by $\gamma$. Portfolios are selected using loss functions indexed by $\gamma^{*}$ and the indicated sets of characteristics. The first in-sample period is January 1960 - December 1974. This is updated annually-and a new portfolio rule is determined at the beginning of each out-of-sample year. The out-of-sample period consists of the 528 months from January 1975 through December 2018. The global optimal portfolios for each loss function are highlighted in boldface. The rolling protocol keeps only the most recent 180 months of data to estimate the characteristic coefficients at the beginning of each year. Under the updating protocol, the last in-sample period comprises 696 months of return data. Characteristic abbreviations are: $\mathrm{M}$ - momentum; $V_{-f}$ - book-to-market ratio exempting financial stocks; $\mathrm{S}$ - $\log$ size; $\beta$ - market model regression coefficient estimated over previous 60 months; $r_{t-12}$ - last year's same-month return; $\bar{r}$ - average same-month return from previous five years; $\sigma_{\epsilon}$ - residual standard deviation from the market model regression estimated over the previous 60 months.

\begin{tabular}{crrrrrr} 
Panel A: $\gamma$ 2 loss function \\
$\begin{array}{c}\text { Characteristics: } \\
\gamma^{*}\end{array}$ & \multicolumn{2}{c}{ V, S, $\beta, r_{t-12}, \bar{r}, \sigma_{\epsilon}$} & \multicolumn{3}{c}{ M, S, $\beta, \bar{r}, \sigma_{\epsilon}$} \\
Updating & $2.5 \%$ ile & Mean & $97.5 \%$ ile & \multicolumn{3}{c}{ Resampled } \\
2 & $-10,000.0$ & $-9,446.3$ & 0.2 & $-10,000.0$ & $-4,212.2$ & 295.8 \\
3 & $-10,000.0$ & $-6,383.9$ & 227.3 & $\mathbf{2 5 7 . 4}$ & $\mathbf{3 0 1 . 6}$ & $\mathbf{3 5 1 . 7}$ \\
4 & $-10,000.0$ & $-1,804.8$ & 250.2 & 255.1 & 293.8 & 336.4 \\
5 & $-10,000.0$ & -111.3 & 247.0 & 240.5 & 275.0 & 313.4 \\
6 & 114.5 & 178.0 & 238.8 & 226.2 & 256.8 & 291.3 \\
7 & 158.4 & 196.2 & 229.2 & 214.2 & 241.0 & 272.8 \\
8 & 164.2 & 192.0 & 218.9 & 202.8 & 228.3 & 256.4 \\
9 & 162.5 & 186.5 & 211.4 & 193.3 & 217.0 & 243.3 \\
10 & 160.3 & 181.0 & 203.5 & 185.2 & 207.2 & 232.2 \\
11 & 156.5 & 176.0 & 197.2 & 177.9 & 198.7 & 222.0 \\
12 & 152.6 & 171.2 & 191.1 & 171.7 & 191.3 & 213.6 \\
Rolling & & & & & & \\
2 & $-10,000.0$ & $-8,693.4$ & 17.7 & $-10,000.0$ & $-6,641.3$ & 176.9 \\
3 & $-10,000.0$ & $-5,253.9$ & 245.3 & $-10,000.0$ & -443.6 & 336.8 \\
4 & $-10,000.0$ & $-2,039.6$ & 276.0 & 143.6 & 172.6 & 344.8 \\
5 & $-10,000.0$ & -424.1 & 271.5 & 223.7 & 269.5 & 325.2 \\
6 & -42.0 & 78.5 & 261.5 & 224.6 & 263.2 & 303.3 \\
7 & 139.2 & 185.5 & 249.5 & 215.6 & 249.1 & 284.6 \\
8 & 159.7 & 196.9 & 238.0 & 205.0 & 235.2 & 267.2 \\
9 & 163.5 & 196.2 & 227.8 & 195.7 & 223.4 & 253.0 \\
10 & 161.3 & 190.1 & 218.6 & 188.3 & 213.0 & 240.4 \\
11 & 158.2 & 184.3 & 210.6 & 179.7 & 203.5 & 229.0 \\
12 & 154.7 & 178.7 & 203.4 & 173.5 & 195.5 & 219.5 \\
\hline
\end{tabular}




\section{Table 2 -Continued}

Estimation Risk: Sampling distribution of out-of-sample certainty equivalent returns (in basis points per month) - as portfolio rules vary

Panel B: $\gamma 5$ loss function

\begin{tabular}{|c|c|c|c|c|c|c|}
\hline $\begin{array}{c}\text { Characteristics: } \\
\gamma^{*}\end{array}$ & \multicolumn{3}{|c|}{$\begin{array}{c}\mathrm{M}, \mathrm{S}, \beta, \bar{r}, \sigma_{\epsilon} \\
\text { Resampled }\end{array}$} & \multicolumn{3}{|c|}{$\begin{array}{l}\mathrm{M}, \mathrm{S}, \bar{r}, \sigma_{\epsilon} \\
\text { Resampled }\end{array}$} \\
\hline Updating & $2.5 \%$ ile & Mean & $97.5 \%$ ile & $2.5 \%$ ile & Mean & $97.5 \%$ ile \\
\hline 5 & 72.3 & 121.5 & 158.6 & 50.1 & 108.5 & 145.7 \\
\hline 6 & 111.0 & 144.4 & 172.9 & 98.4 & 136.5 & 162.8 \\
\hline 7 & 125.9 & 152.5 & 177.4 & 120.7 & 148.2 & 170.9 \\
\hline 8 & 130.3 & 154.2 & 176.7 & 129.8 & 152.6 & 173.3 \\
\hline 9 & 130.9 & 153.0 & 174.5 & 132.2 & 153.1 & 172.5 \\
\hline 10 & 129.8 & 150.6 & 171.1 & 132.7 & 151.9 & 170.2 \\
\hline 11 & 127.5 & 147.3 & 167.5 & 131.9 & 150.1 & 168.0 \\
\hline \multicolumn{7}{|l|}{ Rolling } \\
\hline 5 & $-1,190.3$ & -57.7 & 135.2 & -2432.2 & -165.1 & 133.0 \\
\hline 6 & -341.7 & 67.7 & 165.3 & -531.0 & 35.9 & 159.3 \\
\hline 7 & -92.8 & 115.1 & 176.4 & -132.7 & 101.8 & 169.5 \\
\hline 8 & 13.1 & 132.0 & 178.7 & -1.5 & 126.3 & 172.6 \\
\hline 9 & 52.9 & 137.0 & 176.9 & 47.7 & 135.7 & 171.7 \\
\hline 10 & 68.1 & 138.1 & 173.1 & 72.5 & 138.3 & 170.3 \\
\hline 11 & 76.0 & 136.3 & 168.8 & 86.0 & 138.4 & 167.2 \\
\hline 12 & 82.3 & 133.9 & 164.7 & 91.0 & 136.9 & 164.1 \\
\hline 13 & 84.6 & 130.9 & 159.7 & 91.3 & 134.3 & 160.1 \\
\hline 16 & 83.8 & 120.9 & 146.8 & 90.6 & 126.5 & 151.1 \\
\hline 22 & 75.5 & 103.7 & 126.8 & 72.9 & 109.5 & 134.4 \\
\hline
\end{tabular}

Panel C: $\gamma 9$ loss function

Characteristics: $\quad \mathrm{M}, \mathrm{S}, \bar{r}, \sigma_{\epsilon}$ $\gamma^{*} \quad$ Resampled

Updating

9

10

11

12

13

16

22

Rolling

\begin{tabular}{rrr}
$2.5 \%$ ile & Mean & $97.5 \%$ ile \\
\hline-49.1 & 41.0 & 80.4 \\
-18.7 & 53.3 & 88.0 \\
-1.6 & 61.2 & 92.6 \\
9.7 & 65.8 & 95.5 \\
13.3 & 72.4 & 96.4 \\
19.2 & 68.6 & 95.9 \\
1.5 & 57.4 & 87.8
\end{tabular}

$\begin{array}{lll}-959.0 & -104.2 & 71.9\end{array}$

$\begin{array}{lll}-659.7 & -56.3 & 81.0\end{array}$

$-467.2-28.3$

84.9

$\begin{array}{lll}-368.0 & -11.5 & 87.7\end{array}$

$\begin{array}{lll}-337.7 & -4.3 & 87.4\end{array}$

$\begin{array}{lll}-236.4 & 7.5 & 85.4\end{array}$

$\begin{array}{lll}-247.1 & -8.3 & 73.3\end{array}$

$\mathrm{M}, \mathrm{S}, \sigma_{\epsilon}$ Resampled

\begin{tabular}{rrr}
$2.5 \%$ ile & Mean & $97.5 \%$ ile \\
\hline-50.0 & 12.8 & 46.7 \\
-10.4 & 32.2 & 58.2 \\
9.5 & 43.9 & 65.9 \\
23.9 & 51.6 & 70.6 \\
33.5 & 56.6 & 73.8 \\
$\mathbf{4 4 . 2}$ & $\mathbf{6 2 . 2}$ & $\mathbf{7 7 . 4}$ \\
37.6 & 57.8 & 74.3
\end{tabular}

$\begin{array}{lll}-84.8 & 10.0 & 49.7\end{array}$

$\begin{array}{lll}-38.6 & 27.4 & 57.4\end{array}$

$\begin{array}{lll}-7.9 & 37.3 & 61.6\end{array}$

$\begin{array}{lll}3.9 & 42.4 & 64.0\end{array}$

$\begin{array}{lll}14.2 & 45.2 & 65.4\end{array}$

$\begin{array}{lll}19.5 & 44.4 \quad 63.5\end{array}$

$\begin{array}{lll}-6.1 & 28.3 \quad 52.5\end{array}$ 


\section{Table 3}

\section{Sampling distributions of out-of-sample certainty equivalent returns, portfolio moments and properties}

$\mathcal{C} \mathcal{E}_{i}$ is the certainty equivalent return for a power utility function with coefficient of risk aversion $\gamma=i$, in basis points per month. Feasible portfolio rules are obtained by fixing $\gamma^{*}$, the set of characteristics, and the updating protocol, and then maximizing the following using $T$ months of sample data: $\max _{\theta} \sum_{t=0}^{T-1} \frac{\left(1+r_{p, t+1}\right)^{1-\gamma^{*}}}{1-\gamma^{*}}\left(\frac{1}{T}\right)$ by allowing portfolio weights to depend on observable stock characteristics: $r_{p, t+1}=\sum_{i=1}^{N_{t}}\left(\bar{\omega}_{i, t}+\frac{1}{N_{t}} \theta^{\prime} x_{i, t}\right) \cdot r_{i, t+1}$, where: $x_{i, t}$ is the $K$-vector of characteristics on firm $i$, measurable at time $t ; \bar{\omega}_{i, t}$ is the weight of stock $i$ in the (value-weighted) market portfolio at time $t$; and $N_{t}$ is the number of stocks in the sample at time $t$. Intq Rng $=r_{.75}-r_{.25}$; Skewness $=\frac{\mu-r_{.5}}{\sigma} ;$ Kurtosis $=\frac{\bar{r}_{.95}^{+}-\bar{r}_{.05}^{-}}{\bar{r}_{.5}^{+}-\bar{r}_{.5}^{-}}-2.63 ; \bar{r}_{.95}^{+}$is the mean of the highest $5 \%$ of returns, $\bar{r}_{.05}^{-}$is the mean of the smallest $5 \%$ of returns, $\bar{r}_{.5}^{+}$is the mean of the top half of returns, $\bar{r}_{.5}^{-}$is the mean of the bottom half of returns; and $r_{x}$ is the observation corresponding to the $x \%$ ile of the return data. S.R. (ann.) is the Sharpe ratio of the monthly excess returns multiplied by $\sqrt{12}$. VWI and EWI are the value-weighted and equally-weighted portfolios of sample stocks. The out-of-sample period contains 528 monthly returns-- January 1975 through December 2018.

\section{Panel A: Benchmarks}

\begin{tabular}{|c|c|c|c|c|c|c|}
\hline \multirow[t]{2}{*}{ Metric } & \multicolumn{3}{|c|}{$\begin{array}{l}\text { Resampled } \\
\text { Out of sample }\end{array}$} & \multicolumn{3}{|c|}{$\begin{array}{l}\text { Resampled } \\
\text { Out of sample }\end{array}$} \\
\hline & $2.5 \%$ ile & Mean & $97.5 \%$ ile & $2.5 \%$ ile & Mean & $97.5 \%$ ile \\
\hline Portfolio: & & VWI & & & EWI & \\
\hline $\mathcal{C} \mathcal{E}_{2}$ & 82.5 & 86.7 & 91.0 & 100.6 & 102.7 & 104.8 \\
\hline $\mathcal{C} \mathcal{E}_{5}$ & 51.6 & 56.2 & 60.8 & 57.1 & 59.3 & 61.6 \\
\hline $\mathcal{C E}_{9}$ & 4.4 & 9.8 & 15.2 & -13.4 & -10.3 & -7.2 \\
\hline$E(r)$ & 1.02 & 1.06 & 1.10 & 1.27 & 1.29 & 1.31 \\
\hline$E(r)-r_{f}$ & 0.65 & 0.69 & 0.73 & 0.90 & 0.92 & 0.94 \\
\hline$\sigma(r)$ & 4.27 & 4.31 & 4.37 & 5.04 & 5.07 & 5.09 \\
\hline Median & 1.21 & 1.33 & 1.44 & 1.43 & 1.52 & 1.62 \\
\hline IQ Range & 5.04 & 5.23 & 5.42 & 5.90 & 6.05 & 6.19 \\
\hline Minimum & -23.30 & -22.27 & -21.25 & -26.49 & -25.91 & -25.32 \\
\hline Skewness & -0.09 & -0.06 & -0.04 & -0.06 & -0.04 & -0.03 \\
\hline Kurtosis & 0.31 & 0.36 & 0.41 & 0.36 & 0.38 & 0.40 \\
\hline S.R. (ann.) & 0.52 & 0.55 & 0.59 & 0.61 & 0.63 & 0.64 \\
\hline
\end{tabular}


Table 3 -Continued

Sampling distributions of out-of-sample certainty equivalent returns, portfolio moments and properties

Panel B: $\gamma^{*}=3: \gamma 2$ loss function's perspective

\begin{tabular}{|c|c|c|c|c|c|c|}
\hline \multirow[t]{2}{*}{ Metric } & \multicolumn{3}{|c|}{$\begin{array}{c}\text { Resampled } \\
\text { Out of sample }\end{array}$} & \multicolumn{3}{|c|}{$\begin{array}{c}\text { Resampled } \\
\text { Out of sample }\end{array}$} \\
\hline & $2.5 \%$ ile & Mean & $97.5 \%$ ile & $2.5 \%$ ile & Mean & $97.5 \%$ ile \\
\hline \multirow{2}{*}{$\begin{array}{l}\text { Portfolio : } \\
\mathcal{C E}_{2}\end{array}$} & \multicolumn{3}{|c|}{$\mathrm{M}, \mathrm{S}, \boldsymbol{\beta}, \overline{\boldsymbol{r}}, \sigma_{\epsilon}$} & \multicolumn{3}{|c|}{$\mathrm{S}, \beta, \bar{r}, \sigma_{\epsilon}$} \\
\hline & 257.4 & 301.6 & 351.7 & $-10,000.0$ & $-1,139.4$ & 278.9 \\
\hline$E(r)$ & 4.34 & 5.11 & 6.00 & 3.58 & 4.22 & 4.95 \\
\hline$\sigma(r)$ & 12.40 & 14.11 & 16.10 & 11.57 & 13.06 & 14.71 \\
\hline Median & 4.26 & 5.18 & 6.19 & 3.46 & 4.25 & 5.10 \\
\hline IQ Range & 14.37 & 16.71 & 19.35 & 12.45 & 14.35 & 16.45 \\
\hline Minimum $r$ & -68.33 & -53.56 & -43.16 & -115.01 & -79.65 & -47.53 \\
\hline Skewness & -0.05 & -0.01 & 0.04 & -0.05 & 0.00 & 0.04 \\
\hline Kurtosis & 0.28 & 0.41 & 0.55 & 0.41 & 0.56 & 0.71 \\
\hline S.R. (ann.) & 1.07 & 1.17 & 1.27 & 0.92 & 1.02 & 1.12 \\
\hline Neg.Wts. & -599.2 & -515.4 & -441.4 & -557.1 & -485.8 & -419.9 \\
\hline \multirow{2}{*}{$\begin{array}{l}\text { Portfolio : } \\
\quad \mathcal{C} \mathcal{E}_{2}\end{array}$} & \multicolumn{3}{|c|}{$\mathrm{M}, \beta, \bar{r}, \sigma_{\epsilon}$} & \multicolumn{3}{|c|}{$\mathrm{M}, \mathrm{S}, \bar{r}, \sigma_{\epsilon}$} \\
\hline & 169.7 & 208.0 & 248.2 & 238.5 & 279.2 & 320.6 \\
\hline$E(r)$ & 3.11 & 3.76 & 4.48 & 4.19 & 4.86 & 5.62 \\
\hline$\sigma(r)$ & 10.99 & 12.64 & 14.52 & 12.33 & 13.95 & 15.74 \\
\hline Median & 2.53 & 3.23 & 4.02 & 4.16 & 4.97 & 5.85 \\
\hline IQ Range & 12.04 & 14.07 & 16.37 & 13.78 & 15.75 & 17.89 \\
\hline Minimum $r$ & -67.95 & -54.11 & -42.98 & -68.29 & -54.10 & -44.23 \\
\hline Skewness & 0.00 & 0.04 & 0.08 & -0.05 & -0.01 & 0.04 \\
\hline Kurtosis & 0.43 & 0.57 & 0.72 & 0.39 & 0.52 & 0.65 \\
\hline S.R. (ann.) & 0.83 & 0.93 & 1.03 & 1.03 & 1.12 & 1.21 \\
\hline Neg.Wts. & -479.5 & -400.1 & -329.0 & -569.7 & -493.7 & -423.4 \\
\hline \multirow{3}{*}{$\begin{array}{l}\text { Portfolio: } \\
\mathcal{C E}_{2} \\
E(r)\end{array}$} & \multicolumn{3}{|c|}{$\mathrm{M}, \mathrm{S}, \beta, \sigma_{\epsilon}$} & \multicolumn{3}{|c|}{$\mathrm{M}, \mathrm{S}, \beta, \bar{r}$} \\
\hline & 114.5 & 182.2 & 230.2 & 167.4 & 199.6 & 233.3 \\
\hline & 3.30 & 3.78 & 4.33 & 3.28 & 3.79 & 4.36 \\
\hline$\sigma(r)$ & 11.38 & 12.56 & 13.86 & 11.62 & 12.89 & 14.24 \\
\hline Median & 3.06 & 3.73 & 4.48 & 3.23 & 3.92 & 4.65 \\
\hline IQ Range & 12.04 & 13.62 & 15.43 & 12.81 & 14.49 & 16.26 \\
\hline Minimum $r$ & -86.13 & -67.20 & -51.26 & -62.46 & -51.41 & -43.02 \\
\hline Skewness & -0.04 & 0.00 & 0.04 & -0.05 & -0.01 & 0.03 \\
\hline Kurtosis & 0.50 & 0.63 & 0.76 & 0.38 & 0.51 & 0.65 \\
\hline S.R. (ann.) & 0.86 & 0.94 & 1.03 & 0.84 & 0.92 & 1.01 \\
\hline Neg.Wts. & -422.0 & -366.2 & -317.4 & -506.0 & -443.2 & -387.6 \\
\hline
\end{tabular}


Table 3 -Continued

Sampling distributions of out-of-sample certainty equivalent returns, portfolio moments and properties

\begin{tabular}{|c|c|c|c|c|c|c|}
\hline \multirow[t]{2}{*}{ Metric } & \multicolumn{3}{|c|}{$\begin{array}{c}\text { Resampled } \\
\text { Out of sample }\end{array}$} & \multicolumn{3}{|c|}{$\begin{array}{l}\text { Resampled } \\
\text { Out of sample }\end{array}$} \\
\hline & $2.5 \%$ ile & Mean & $97.5 \%$ ile & $2.5 \%$ ile & Mean & $97.5 \%$ ile \\
\hline \multirow{2}{*}{$\begin{array}{l}\text { Portfolio: } \\
\mathcal{C E}_{2}\end{array}$} & \multicolumn{3}{|c|}{$\mathrm{M}, \mathrm{V}, \mathrm{S}, \beta, \bar{r}, \sigma_{\epsilon}$} & \multicolumn{3}{|c|}{$\mathrm{M}, \mathrm{V}, \beta, \bar{r}, \sigma_{\epsilon}$} \\
\hline & 247.4 & 271.3 & 350.9 & 167.6 & 200.2 & 294.9 \\
\hline$E(r)$ & 4.31 & 5.09 & 6.00 & 3.49 & 4.20 & 5.02 \\
\hline$\sigma(r)$ & 12.29 & 14.10 & 16.21 & 10.99 & 12.81 & 14.92 \\
\hline Median & 4.23 & 5.12 & 6.14 & 3.16 & 3.95 & 4.82 \\
\hline IQ Range & 13.74 & 16.16 & 18.89 & 12.38 & 14.65 & 17.30 \\
\hline Minimum $r$ & -75.30 & -52.75 & -40.13 & -84.54 & -55.33 & -38.39 \\
\hline Skewness & -0.05 & 0.00 & 0.04 & -0.02 & 0.02 & 0.06 \\
\hline Kurtosis & 0.34 & 0.48 & 0.62 & 0.28 & 0.43 & 0.58 \\
\hline S.R. (ann.) & 1.06 & 1.16 & 1.26 & 0.93 & 1.03 & 1.14 \\
\hline Neg.Wts. & -583.7 & -496.9 & -420.5 & -512.5 & -431.1 & -358.7 \\
\hline
\end{tabular}

Panel C: $\gamma^{*}=10: \gamma 5$ loss function's perspective

\begin{tabular}{|c|c|c|c|c|c|c|}
\hline \multirow{2}{*}{$\begin{array}{l}\text { Portfolio: } \\
\mathcal{C E}_{5}\end{array}$} & \multicolumn{3}{|c|}{$\mathrm{M}, \mathrm{S}, \bar{r}, \sigma_{\epsilon}$} & \multicolumn{3}{|c|}{$\mathrm{S}, \bar{r}, \sigma_{\epsilon}$} \\
\hline & 132.7 & 151.9 & 170.2 & 56.5 & 116.2 & 146.5 \\
\hline$E(r)$ & 2.20 & 2.44 & 2.71 & 1.98 & 2.18 & 2.41 \\
\hline$\sigma(r)$ & 5.33 & 5.82 & 6.36 & 5.21 & 5.62 & 6.08 \\
\hline Median & 2.32 & 2.64 & 2.97 & 2.02 & 2.30 & 2.59 \\
\hline IQ Range & 5.57 & 6.22 & 6.95 & 5.05 & 5.61 & 6.22 \\
\hline Minimum $r$ & -37.75 & -27.35 & -20.12 & -53.51 & -40.88 & -29.22 \\
\hline Skewness & -0.07 & -0.03 & 0.00 & -0.06 & -0.02 & 0.02 \\
\hline Kurtosis & 0.52 & 0.64 & 0.77 & 0.58 & 0.71 & 0.85 \\
\hline S.R. (ann.) & 1.15 & 1.24 & 1.32 & 1.03 & 1.12 & 1.21 \\
\hline Neg.Wts. & -168.0 & -144.3 & -122.8 & -152.8 & -131.0 & -111.4 \\
\hline \multirow{2}{*}{$\begin{array}{l}\text { Portfolio: } \\
\mathcal{C E}_{5}\end{array}$} & \multicolumn{3}{|c|}{$\mathrm{M}, \bar{r}, \sigma_{\epsilon}$} & \multicolumn{3}{|c|}{$\mathrm{M}, \mathrm{S}, \sigma_{\epsilon}$} \\
\hline & 92.3 & 107.6 & 121.7 & 110.5 & 123.4 & 135.9 \\
\hline$E(r)$ & 1.62 & 1.79 & 1.98 & 1.90 & 2.05 & 2.21 \\
\hline$\sigma(r)$ & 4.81 & 5.19 & 5.62 & 5.06 & 5.37 & 5.72 \\
\hline Median & 1.48 & 1.71 & 1.95 & 1.96 & 2.20 & 2.43 \\
\hline IQ Range & 4.86 & 5.34 & 5.86 & 5.08 & 5.53 & 6.02 \\
\hline Minimum $r$ & -36.51 & -26.55 & -18.26 & -35.13 & -28.02 & -22.33 \\
\hline Skewness & -0.02 & 0.02 & 0.05 & -0.06 & -0.03 & 0.01 \\
\hline Kurtosis & 0.55 & 0.67 & 0.80 & 0.58 & 0.68 & 0.79 \\
\hline S.R. (ann.) & 0.87 & 0.95 & 1.03 & 1.02 & 1.09 & 1.16 \\
\hline Neg.Wts. & -127.1 & -107.9 & -91.1 & -120.6 & -107.7 & -96.1 \\
\hline
\end{tabular}


Table 3 -Continued

Sampling distributions of out-of-sample certainty equivalent returns, portfolio moments and properties

\begin{tabular}{|c|c|c|c|c|c|c|}
\hline \multirow[t]{2}{*}{ Metric } & \multicolumn{3}{|c|}{$\begin{array}{l}\text { Resampled } \\
\text { Out of sample }\end{array}$} & \multicolumn{3}{|c|}{$\begin{array}{c}\text { Resampled } \\
\text { Out of sample }\end{array}$} \\
\hline & $2.5 \%$ ile & Mean & $97.5 \%$ ile & $2.5 \%$ ile & Mean & $97.5 \%$ ile \\
\hline Portfolio: & \multicolumn{3}{|c|}{$\mathrm{M}, \mathrm{S}, \bar{r}$} & \multicolumn{3}{|c|}{$\mathrm{M}, \mathrm{V}, \mathrm{S}, \bar{r}, \sigma_{\epsilon}$} \\
\hline $\mathcal{C} \mathcal{E}_{5}$ & 58.4 & 65.6 & 73.2 & 121.8 & 146.7 & 166.7 \\
\hline$E(r)$ & 1.31 & 1.42 & 1.53 & 2.20 & 2.44 & 2.70 \\
\hline$\sigma(r)$ & 5.09 & 5.31 & 5.54 & 5.42 & 5.92 & 6.46 \\
\hline Median & 1.39 & 1.58 & 1.77 & 2.30 & 2.61 & 2.94 \\
\hline IQ Range & 5.40 & 5.71 & 6.03 & 5.60 & 6.25 & 6.94 \\
\hline Minimum $r$ & -25.38 & -23.72 & -22.12 & -42.87 & -31.24 & -22.05 \\
\hline Skewness & -0.06 & -0.03 & 0.00 & -0.07 & -0.03 & 0.01 \\
\hline Kurtosis & 0.51 & 0.58 & 0.66 & 0.54 & 0.66 & 0.78 \\
\hline S.R. (ann.) & 0.63 & 0.68 & 0.74 & 1.13 & 1.22 & 1.31 \\
\hline Neg.Wts. & -65.4 & -52.6 & -41.3 & -163.4 & -140.9 & -120.0 \\
\hline Portfolio: & \multicolumn{3}{|c|}{$\mathrm{M}, \mathrm{S}, \beta, \bar{r}, \sigma_{\epsilon}$} & \multicolumn{3}{|c|}{$\sigma_{\epsilon}$} \\
\hline $\mathcal{C} \mathcal{E}_{5}$ & 129.8 & 150.6 & 171.1 & 66.2 & 74.2 & 81.9 \\
\hline$E(r)$ & 2.16 & 2.42 & 2.72 & 1.10 & 1.16 & 1.23 \\
\hline$\sigma(r)$ & 5.29 & 5.80 & 6.37 & 3.83 & 3.95 & 4.07 \\
\hline Median & 2.32 & 2.62 & 2.95 & 1.05 & 1.20 & 1.34 \\
\hline IQ Range & 5.51 & 6.23 & 7.04 & 4.00 & 4.23 & 4.47 \\
\hline Minimum $r$ & -37.16 & -26.76 & -19.73 & -29.39 & -24.22 & -19.34 \\
\hline Skewness & -0.08 & -0.03 & 0.01 & -0.04 & -0.01 & 0.03 \\
\hline Kurtosis & 0.49 & 0.62 & 0.74 & 0.49 & 0.57 & 0.66 \\
\hline S.R. (ann.) & 1.13 & 1.23 & 1.33 & 0.63 & 0.70 & 0.76 \\
\hline Neg.Wts. & -173.3 & -148.1 & -125.6 & -51.4 & -47.1 & -43.1 \\
\hline
\end{tabular}


Table 3 -Continued

Sampling distributions of out-of-sample certainty equivalent returns, portfolio moments and properties

Metric Resampled

Out of sample
Resampled

Out of sample

Panel D: $\gamma^{*}=16: \gamma 9$ loss function's perspective

\begin{tabular}{|c|c|c|c|c|c|c|}
\hline \multirow{2}{*}{$\begin{array}{l}\text { Portfolio: } \\
\qquad \mathcal{C E}_{9}\end{array}$} & \multicolumn{3}{|c|}{$\mathrm{M}, \mathrm{S}, \sigma_{\epsilon}$} & \multicolumn{3}{|c|}{$\mathrm{S} \sigma_{\epsilon}$} \\
\hline & 44.2 & 62.2 & 77.4 & 25.2 & 47.3 & 62.9 \\
\hline$E(r)$ & 1.64 & 1.76 & 1.89 & 1.33 & 1.42 & 1.51 \\
\hline$\sigma(r)$ & 4.33 & 4.54 & 4.77 & 3.96 & 4.12 & 4.29 \\
\hline Median & 1.70 & 1.89 & 2.08 & 1.27 & 1.41 & 1.56 \\
\hline IQ Range & 4.33 & 4.66 & 5.02 & 3.80 & 4.07 & 4.35 \\
\hline Minimum $r$ & -27.55 & -22.29 & -18.44 & -30.61 & -24.83 & -19.47 \\
\hline Skewness & -0.06 & -0.03 & 0.01 & -0.03 & 0.00 & 0.04 \\
\hline Kurtosis & 0.58 & 0.68 & 0.78 & 0.67 & 0.77 & 0.87 \\
\hline S.R. (ann.) & 0.99 & 1.07 & 1.14 & 0.81 & 0.88 & 0.95 \\
\hline Neg.Wts. & -85.6 & -77.0 & -69.0 & -59.8 & -53.5 & -47.4 \\
\hline Portfolio: & \multicolumn{3}{|c|}{$\mathrm{M}, \sigma_{\epsilon}$} & \multicolumn{3}{|c|}{$\mathrm{M}, \mathrm{S}$} \\
\hline $\mathcal{C} \mathcal{E}_{9}$ & 32.6 & 45.0 & 56.1 & -4.4 & 2.8 & 9.8 \\
\hline$E(r)$ & 1.25 & 1.33 & 1.41 & 1.00 & 1.06 & 1.12 \\
\hline$\sigma(r)$ & 3.96 & 4.09 & 4.22 & 4.40 & 4.47 & 4.55 \\
\hline Median & 1.24 & 1.40 & 1.56 & 1.03 & 1.18 & 1.33 \\
\hline IQ Range & 4.19 & 4.44 & 4.70 & 4.90 & 5.14 & 5.37 \\
\hline Minimum $r$ & -24.48 & -20.30 & -16.73 & -23.86 & -22.59 & -21.30 \\
\hline Skewness & -0.05 & -0.02 & 0.02 & -0.06 & -0.03 & 0.00 \\
\hline Kurtosis & 0.47 & 0.56 & 0.65 & 0.33 & 0.39 & 0.44 \\
\hline S.R. (ann.) & 0.75 & 0.81 & 0.88 & 0.49 & 0.53 & 0.58 \\
\hline Neg.Wts. & -62.0 & -57.4 & -53.1 & -35.4 & -30.3 & -25.2 \\
\hline Portfolio: & \multicolumn{3}{|c|}{$\mathrm{M}, V_{-f}, \mathrm{~S}, \sigma_{\epsilon}$} & \multicolumn{3}{|c|}{$\mathrm{M}, \mathrm{S}, \beta, \bar{r}, \sigma_{\epsilon}$} \\
\hline $\mathcal{C} \mathcal{E}_{9}$ & 36.5 & 57.4 & 74.3 & 13.1 & 61.3 & 89.7 \\
\hline$E(r)$ & 1.65 & 1.77 & 1.90 & 1.74 & 1.92 & 2.13 \\
\hline$\sigma(r)$ & 4.44 & 4.66 & 4.89 & 4.41 & 4.72 & 5.07 \\
\hline Median & 1.72 & 1.91 & 2.11 & 1.94 & 2.16 & 2.39 \\
\hline IQ Range & 4.45 & 4.80 & 5.16 & 4.41 & 4.84 & 5.33 \\
\hline Minimum $r$ & -29.11 & -23.54 & -19.08 & -35.73 & -27.59 & -20.14 \\
\hline Skewness & -0.07 & -0.03 & 0.00 & -0.09 & -0.05 & -0.01 \\
\hline Kurtosis & 0.56 & 0.67 & 0.77 & 0.56 & 0.68 & 0.81 \\
\hline S.R. (ann.) & 0.97 & 1.05 & 1.12 & 1.05 & 1.15 & 1.25 \\
\hline Neg.Wts. & -86.0 & -77.7 & -70.1 & -109.0 & -92.8 & -78.8 \\
\hline
\end{tabular}




\section{Table 4}

\section{Sampling distribution of $\theta$ coefficients on characteristics}

Feasible portfolio rules are obtained by fixing $\gamma^{*}$, the set of characteristics, and the updating protocol, and then maximizing the following using $T$ months of sample data: $\max _{\theta} \sum_{t=0}^{T-1} \frac{\left(1+r_{p, t+1}\right)^{1-\gamma^{*}}}{1-\gamma^{*}}\left(\frac{1}{T}\right)$ by allowing portfolio weights to depend on observable stock characteristics:

$r_{p, t+1}=\sum_{i=1}^{N_{t}}\left(\bar{\omega}_{i, t}+\frac{1}{N_{t}} \theta^{\prime} x_{i, t}\right) \cdot r_{i, t+1}$, where: $x_{i, t}$ is the $K$-vector of characteristics on firm $i$, measurable at time $t ; \bar{\omega}_{i, t}$ is the weight of stock $i$ in the (value-weighted) market portfolio at time $t$; and $N_{t}$ is the number of stocks in the sample at time $t$. The $\theta$-vector is updated annually, (and applied to the next 12 months of out-of-sample returns, to construct 528 monthly out-of-sample returns). This generates 44 (annual) $\theta$-vectors. This table reports small sample properties of the mean of these 44 vectors for 30 different feasible portfolio rules: characteristic set and curvature of loss function $\left(\gamma^{*}\right)$ under the updating protocol (from 10,000 pseudosamples). Characteristic abbreviations are: $\mathrm{M}$ - momentum; $V$ - book-to-market ratio (all stocks); $V_{-f}$ - book-to-market ratio exempting financial stocks; $\mathrm{S}$ - $\log$ size; $\beta$ - market model regression coefficient estimated over previous 60 months; $r_{t-12}$ - last year's same-month return; $\bar{r}$ - average same-month return from previous five years; $\sigma_{\epsilon}$ - residual standard deviation from the market model regression estimated over the previous 60 months.

Panel A: $\gamma^{*}=3$. Emboldened portfolio optimal for $\gamma 2$ investor.

\begin{tabular}{|c|c|}
\hline Resampled & Resampled \\
\hline Mo & \\
\hline
\end{tabular}

\begin{tabular}{|c|c|c|c|c|c|c|}
\hline \multirow{2}{*}{$\begin{array}{c}\text { Characteristics: } \\
\text { M }\end{array}$} & \multicolumn{3}{|c|}{$\mathrm{M}, \mathrm{S}, \boldsymbol{\beta}, \overline{\boldsymbol{r}}, \sigma_{\epsilon}$} & \multicolumn{3}{|c|}{$\mathrm{S}, \beta, \bar{r}, \sigma_{\epsilon}$} \\
\hline & 3.65 & 5.31 & 7.04 & & & \\
\hline $\mathrm{S}$ & -14.09 & -10.62 & -7.84 & -13.21 & -9.88 & -7.09 \\
\hline$\beta$ & -1.64 & 3.59 & 7.52 & -5.05 & 0.43 & 4.45 \\
\hline $\bar{r}$ & 6.26 & 9.20 & 12.13 & 7.66 & 11.06 & 14.64 \\
\hline$\sigma_{\epsilon}$ & -15.95 & -11.88 & -6.80 & -11.39 & -7.77 & -3.33 \\
\hline
\end{tabular}

\begin{tabular}{crrrrrrr} 
Characteristics: & \multicolumn{3}{c}{$\mathrm{M}, \beta, \bar{r}, \sigma_{\epsilon}$} & & \multicolumn{3}{c}{$\mathrm{M}, \mathrm{S}, \bar{r}, \sigma_{\epsilon}$} \\
\cline { 2 - 4 } \cline { 6 - 8 } $\mathrm{M}$ & 3.16 & 4.80 & 6.43 & & 2.88 & 4.85 & 6.70 \\
$\mathrm{~S}$ & & & & & -14.31 & -10.82 & -7.96 \\
$\beta$ & 2.89 & 5.77 & 8.80 & & & & \\
$\bar{r}$ & 5.81 & 8.32 & 11.87 & & 6.24 & 9.20 & 12.05 \\
$\sigma_{\epsilon}$ & -13.06 & -9.50 & -5.88 & & -11.03 & -8.17 & -5.83 \\
\hline
\end{tabular}

\begin{tabular}{crrrrrrr} 
Characteristics: & \multicolumn{3}{c}{$\mathrm{M}, \mathrm{S}, \beta, \sigma_{\epsilon}$} & & \multicolumn{3}{c}{$\mathrm{M}, \mathrm{S}, \beta, \bar{r}$} \\
\cline { 2 - 4 } & 3.95 & 6.32 & 8.30 & & 1.79 & 3.74 & 5.41 \\
$\mathrm{~S}$ & -12.59 & -9.02 & -6.17 & & -13.15 & -8.82 & -5.76 \\
$\beta$ & -1.58 & 3.43 & 6.98 & & -9.12 & -6.17 & -3.67 \\
$\bar{r}$ & & & & & 6.39 & 8.88 & 11.54 \\
$\sigma_{\epsilon}$ & -14.26 & -10.53 & -6.20 & & & & \\
\hline
\end{tabular}




\section{Table 4 -Continued}

Sampling distribution of $\theta$ coefficients on characteristics

\begin{tabular}{crrrrrrr} 
& \multicolumn{3}{c}{ Resampled } & & \multicolumn{3}{c}{ Resampled } \\
\cline { 2 - 3 } \cline { 6 - 8 } & $2.5 \%$ ile & Mean & $97.5 \%$ ile & & $2.5 \%$ ile & Mean & $97.5 \%$ ile \\
Characteristics: & \multicolumn{2}{c}{$\mathrm{M}, \mathrm{V}, \mathrm{S}, \beta, \bar{r}, \sigma_{\epsilon}$} & & \multicolumn{3}{c}{$\mathrm{M}, \mathrm{V}, \beta, \bar{r}, \sigma_{\epsilon}$} \\
\cline { 2 - 3 } & 4.79 & 6.25 & 7.92 & & 5.71 & 6.78 & 7.89 \\
$\mathrm{~V} / V_{-f}$ & 1.23 & 3.92 & 6.81 & & 5.93 & 7.60 & 9.37 \\
$\mathrm{~S}$ & -12.46 & -8.59 & -5.68 & & & & \\
$\beta$ & -2.24 & 3.41 & 7.43 & & 2.13 & 4.57 & 7.20 \\
$\bar{r}$ & 6.14 & 9.12 & 12.13 & & 6.14 & 8.12 & 10.25 \\
$\sigma_{\epsilon}$ & -14.39 & -10.27 & -4.87 & & -10.38 & -7.23 & -4.30 \\
\hline
\end{tabular}

Panel B: $\gamma^{*}=10$. Emboldened portfolio optimal for $\gamma 5$ investor.

\begin{tabular}{|c|c|c|c|c|c|c|}
\hline \multirow{2}{*}{$\begin{array}{c}\text { Characteristics: } \\
\text { M }\end{array}$} & \multicolumn{3}{|c|}{$\mathrm{M}, \mathrm{S}, \bar{r}, \sigma_{\epsilon}$} & \multicolumn{3}{|c|}{$\mathrm{S}, \bar{r}, \sigma_{\epsilon}$} \\
\hline & 1.17 & 1.77 & 2.47 & & & \\
\hline S & -5.21 & -3.99 & -2.75 & -4.52 & -3.53 & -2.43 \\
\hline $\bar{r}$ & 1.76 & 2.73 & 3.77 & 1.75 & 3.21 & 4.56 \\
\hline$\sigma_{\epsilon}$ & -5.12 & -3.87 & -2.67 & -4.54 & -3.34 & -2.01 \\
\hline \multirow{2}{*}{$\begin{array}{c}\text { Characteristics: } \\
\text { M }\end{array}$} & \multicolumn{3}{|c|}{$\mathrm{M}, \bar{r}, \sigma_{\epsilon}$} & \multicolumn{3}{|c|}{$\mathrm{M}, \mathrm{S}, \sigma_{\epsilon}$} \\
\hline & \multirow[t]{2}{*}{0.96} & \multirow[t]{2}{*}{1.44} & \multirow[t]{2}{*}{1.94} & 1.07 & 1.96 & 2.82 \\
\hline $\mathrm{S}$ & & & & -4.79 & -3.58 & -2.31 \\
\hline $\bar{r}$ & 1.54 & 2.47 & 3.65 & & & \\
\hline$\sigma_{\epsilon}$ & -2.71 & -2.04 & -1.49 & -4.74 & -3.56 & -2.33 \\
\hline \multirow{2}{*}{$\begin{array}{c}\text { Characteristics: } \\
\text { M }\end{array}$} & \multicolumn{3}{|c|}{$\mathrm{M}, \mathrm{S}, \bar{r}$} & \multicolumn{3}{|c|}{$\mathrm{M}, \mathrm{V}, \mathrm{S}, \bar{r}, \sigma_{\epsilon}$} \\
\hline & 0.41 & 0.85 & 1.90 & 1.27 & 1.97 & 2.60 \\
\hline V & & & & -0.10 & 0.86 & 1.95 \\
\hline S & -0.06 & 0.39 & 0.81 & -4.79 & -3.54 & -2.49 \\
\hline $\bar{r}$ & 0.56 & 1.43 & 2.66 & 1.74 & 2.71 & 3.76 \\
\hline$\sigma_{\epsilon}$ & & & & -4.72 & -3.56 & -2.51 \\
\hline
\end{tabular}

Characteristics:

\begin{tabular}{lrrr} 
& \multicolumn{3}{c}{$\mathrm{M}, \mathrm{S}, \beta, \bar{r}, \sigma_{\epsilon}$} \\
\cline { 2 - 4 } $\mathrm{M}$ & 1.27 & 1.79 & 2.45 \\
$\mathrm{~S}$ & -5.31 & -4.04 & -2.75 \\
$\beta$ & -1.51 & 0.09 & 1.53 \\
$\bar{r}$ & 1.80 & 2.76 & 3.78 \\
$\sigma_{\epsilon}$ & -5.37 & -4.03 & -2.70
\end{tabular}

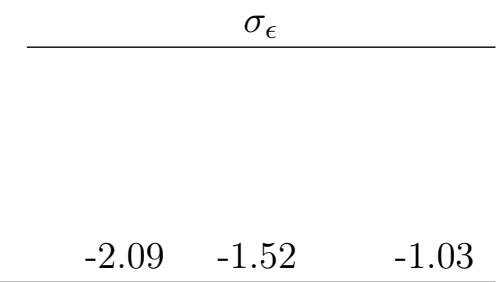

Panel C: $\gamma^{*}=16$. Emboldened portfolio optimal for $\gamma 9$ investor.

Characteristics:

$\mathrm{M}$

$\mathrm{S}$

$\sigma_{\epsilon}$
$\mathrm{M}, \mathrm{S}, \sigma_{\epsilon}$

$\begin{array}{rrr}0.68 & 1.26 & 1.96 \\ -3.44 & -2.46 & -1.38 \\ -3.91 & -2.92 & -1.89\end{array}$

$\mathrm{S}, \sigma_{\epsilon}$

$-2.57 \quad-1.92 \quad-1.23$

$-3.13 \quad-2.30 \quad-1.50$ 


\section{Table 4 -Continued}

\section{Sampling distribution of $\theta$ coefficients on characteristics}

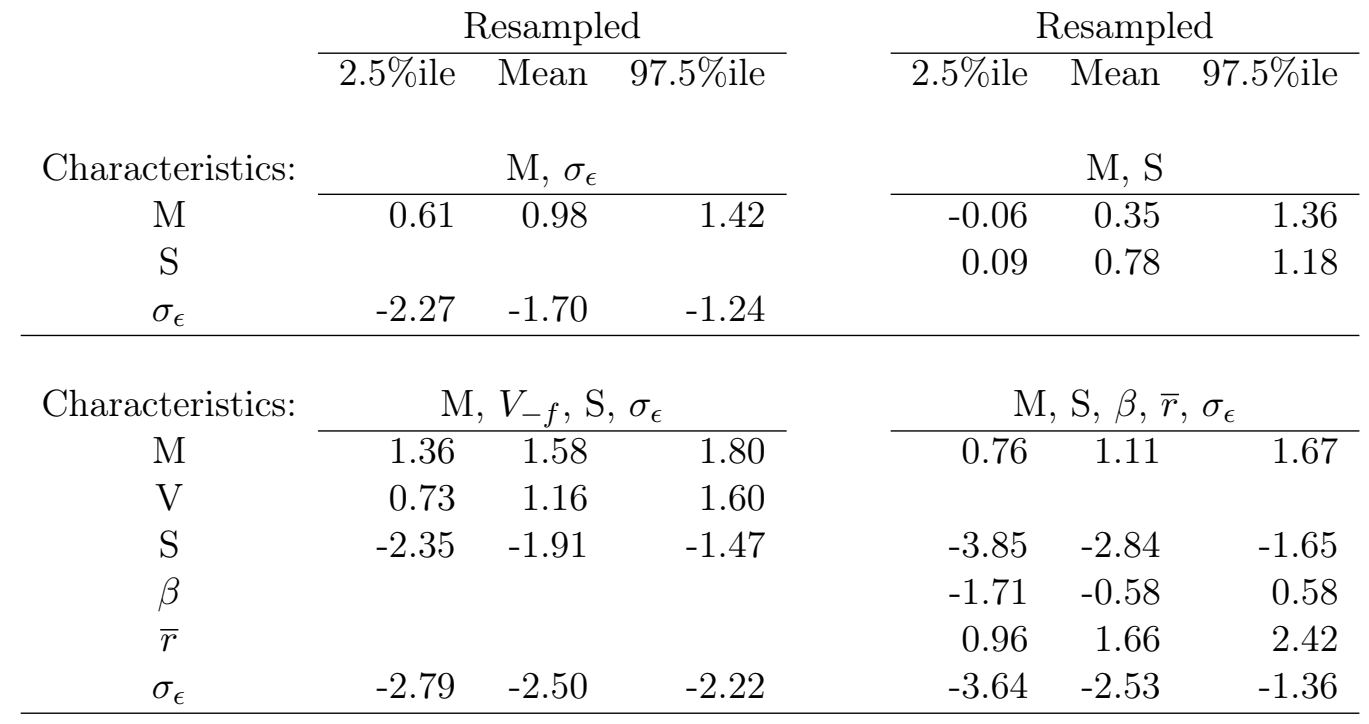

Panel D: $\theta$ coefficient standard-deviation / correlation matrices

$\mathrm{M}, \mathrm{S}, \boldsymbol{\beta}, \overline{\boldsymbol{r}}, \boldsymbol{\sigma}_{\epsilon} ; \gamma^{*}=\mathbf{3}$

Optimal portfolio for $\gamma 2$ loss function

\begin{tabular}{cccccc}
\hline Characteristic: & $\mathrm{M}$ & $\mathrm{S}$ & $\beta$ & $\bar{r}$ & $\sigma_{\epsilon}$ \\
\cline { 1 - 2 } $\mathrm{M}$ & 0.95 & -0.60 & -0.34 & 0.01 & -0.05 \\
$\mathrm{~S}$ & & 1.72 & 0.60 & -0.02 & -0.15 \\
$\beta$ & & & 2.50 & 0.22 & -0.85 \\
$\bar{r}$ & & & & 1.45 & -0.32 \\
$\sigma_{\epsilon}$ & & & & & 2.28 \\
\hline
\end{tabular}

$\mathrm{M}, \mathrm{S}, \bar{r}, \sigma_{\epsilon} ; \gamma^{*}=\mathbf{1 0}$

Optimal portfolio for $\gamma 5$ loss function

\begin{tabular}{ccccc}
\cline { 1 - 4 } Characteristic: & $\mathrm{M}$ & $\mathrm{S}$ & $\bar{r}$ & $\sigma_{\epsilon}$ \\
\cline { 1 - 1 } $\mathrm{M}$ & 0.37 & -0.75 & 0.09 & -0.73 \\
$\mathrm{~S}$ & & 0.71 & -0.26 & 0.92 \\
$\bar{r}$ & & & 0.51 & -0.31 \\
$\sigma_{\epsilon}$ & & & & 0.80 \\
\hline
\end{tabular}

$\mathrm{M}, \mathrm{S}, \sigma_{\epsilon} ; \gamma^{*}=\mathbf{1 6}$

Optimal portfolio for $\gamma 9$ loss function

\begin{tabular}{cccc}
\cline { 1 - 1 } Characteristic: & $\mathrm{M}$ & $\mathrm{S}$ & $\sigma_{\epsilon}$ \\
$\mathrm{yn}$ & 0.40 & -0.87 & -0.80 \\
$\mathrm{~S}$ & & 0.64 & 0.91 \\
$\sigma_{\epsilon}$ & & & 0.71 \\
\hline
\end{tabular}




\section{Table 5}

\section{Decomposition of portfolio expected return and variance based on Fama-French-Carhart Factors}

Panel A reports results from the linear regression:

$r_{p, t}-r_{f, t}=\alpha+\beta_{M} \cdot\left(r_{M, t}-r_{f, t}\right)+\beta_{S} \cdot r_{S, t}+\beta_{H} \cdot r_{H, t}+\beta_{m} \cdot r_{m, t}+\epsilon_{t}$. Where $r_{p, t}$ is the portfolio return in month $t, r_{f, t}$ is the return on one-month Treasury bill in month $t, r_{M, t}$ is the return on the value-weighted market in month $t, r_{S, t}$ is the return on the SMB factor portfolio in month $t$, $r_{H, t}$ is the return on the HML factor portfolio in month $t$, and $r_{m, t}$ is the return on the momentum factor portfolio in month $t$. This decomposes the portfolio variance into 10 (additive) components. We report only covariance terms greater than $5 \%$ in absolute value (so the reported components will not sum to unity). The volatility-correlation matrix in Panel B reports the monthly factor standard deviations along the diagonal and correlations between the factors, off-diagonal. Panel A $\alpha$ values and Panel B factor means are reported in basis points per month.

\section{Panel A. Portfolio factor exposures}

\begin{tabular}{|c|c|c|c|c|c|c|}
\hline \multirow[t]{2}{*}{ Metric } & \multicolumn{3}{|c|}{$\begin{array}{c}\text { Resampled } \\
\text { Out of sample } \\
\text { regression coefficient }\end{array}$} & \multicolumn{3}{|c|}{$\begin{array}{c}\text { Resampled } \\
\text { Out of sample } \\
\text { variance component }(\%)\end{array}$} \\
\hline & $2.5 \%$ ile & Mean & $97.5 \%$ ile & $2.5 \%$ ile & Mean & $97.5 \%$ ile \\
\hline Portfolio: & \multicolumn{6}{|c|}{ Value-weighted portfolio of all sample stocks } \\
\hline$\alpha /$ Orthog. & -2.00 & 2.65 & 7.17 & 1.40 & 1.70 & 2.04 \\
\hline MKT & 0.98 & 0.99 & 1.00 & 101.32 & 102.40 & 103.41 \\
\hline SMB & -0.11 & -0.09 & -0.07 & 0.23 & 0.41 & 0.62 \\
\hline HML & 0.02 & 0.04 & 0.07 & 0.02 & 0.10 & 0.20 \\
\hline MOM & -0.02 & 0.00 & 0.01 & 0.00 & 0.01 & 0.04 \\
\hline Cov(MKT, SMB) & & & & -4.08 & -3.30 & -2.49 \\
\hline Cov(MKT , HML) & & & & -2.22 & -1.46 & -0.71 \\
\hline Portfolio: & \multicolumn{6}{|c|}{ Equally-weighted portfolio of all sample stocks } \\
\hline$\alpha /$ Orthog. & 6.23 & 8.49 & 10.76 & 2.75 & 2.95 & 3.15 \\
\hline MKT & 1.02 & 1.03 & 1.03 & 79.13 & 79.74 & 80.34 \\
\hline SMB & 0.47 & 0.48 & 0.49 & 7.86 & 8.21 & 8.58 \\
\hline HML & 0.25 & 0.26 & 0.27 & 2.05 & 2.22 & 2.39 \\
\hline MOM & -0.09 & -0.08 & -0.07 & 0.37 & 0.46 & 0.56 \\
\hline Cov(MKT , SMB) & & & & 12.79 & 13.04 & 13.29 \\
\hline Cov(MKT , HML) & & & & -6.69 & -6.43 & -6.18 \\
\hline Portfolio: & \multicolumn{6}{|c|}{$\mathrm{M}, \mathrm{S}, \boldsymbol{\beta}, \overline{\boldsymbol{r}}, \sigma_{\epsilon} ; \gamma^{*}=\mathbf{3}$} \\
\hline$\alpha /$ Orthog. & 201.29 & 269.39 & 347.78 & 61.31 & 68.63 & 75.47 \\
\hline MKT & 0.30 & 0.52 & 0.75 & 0.87 & 2.85 & 5.69 \\
\hline SMB & 0.59 & 1.02 & 1.45 & 1.49 & 5.05 & 9.87 \\
\hline HML & 1.61 & 1.99 & 2.39 & 11.92 & 16.60 & 21.36 \\
\hline $\mathrm{MOM}$ & 1.18 & 1.46 & 1.75 & 13.60 & 20.39 & 27.93 \\
\hline Cov(SMB , HML) & & & & -5.47 & -3.61 & -1.87 \\
\hline Cov(HML , MOM) & & & & -9.86 & -8.11 & -6.35 \\
\hline
\end{tabular}


Table 5 -Continued

Decomposition of portfolio expected return and variance based on

Fama-French-Carhart Factors

\begin{tabular}{|c|c|c|c|c|c|c|}
\hline \multirow[t]{2}{*}{ Metric } & \multicolumn{3}{|c|}{$\begin{array}{c}\text { Resampled } \\
\text { Out of sample } \\
\text { regression coefficient }\end{array}$} & \multicolumn{3}{|c|}{$\begin{array}{c}\text { Resampled } \\
\text { Out of sample } \\
\text { variance component }\end{array}$} \\
\hline & $2.5 \%$ ile & Mean & $97.5 \%$ ile & $2.5 \%$ ile & Mean & $97.5 \%$ ile \\
\hline Portfolio: & \multicolumn{6}{|c|}{$\mathrm{S}, \beta, \bar{r}, \sigma_{\epsilon} ; \gamma^{*}=3$} \\
\hline$\alpha /$ Orthog. & 248.66 & 311.30 & 382.04 & 75.94 & 81.15 & 86.13 \\
\hline MKT & 0.23 & 0.43 & 0.63 & 0.58 & 2.23 & 4.63 \\
\hline $\mathrm{SMB}$ & 0.11 & 0.47 & 0.83 & 0.06 & 1.38 & 3.68 \\
\hline HML & 1.49 & 1.79 & 2.11 & 11.49 & 15.70 & 20.07 \\
\hline MOM & -0.53 & -0.32 & -0.12 & 0.16 & 1.23 & 2.83 \\
\hline Cov(MKT , HML) & & & & -4.04 & -2.76 & -1.50 \\
\hline Portfolio: & \multicolumn{6}{|c|}{$\mathrm{M}, \beta, \bar{r}, \sigma_{\epsilon} ; \gamma^{*}=3$} \\
\hline$\alpha /$ Orthog. & 124.60 & 182.27 & 248.00 & 50.07 & 57.72 & 65.06 \\
\hline MKT & 1.18 & 1.35 & 1.52 & 17.16 & 22.36 & 28.06 \\
\hline SMB & -1.52 & -1.19 & -0.88 & 4.76 & 8.11 & 11.82 \\
\hline HML & -0.11 & 0.12 & 0.37 & 0.00 & 0.14 & 0.63 \\
\hline MOM & 1.24 & 1.49 & 1.74 & 18.80 & 26.38 & 34.65 \\
\hline $\operatorname{Cov}(\mathrm{MKT}, \mathrm{SMB})$ & & & & -8.27 & -6.79 & -5.29 \\
\hline $\operatorname{Cov}(\mathrm{MKT}, \mathrm{MOM})$ & & & & -6.24 & -5.25 & -4.29 \\
\hline Portfolio: & \multicolumn{6}{|c|}{$\mathrm{M}, \mathrm{S}, \bar{r}, \sigma_{\epsilon} ; \gamma^{*}=3$} \\
\hline$\alpha /$ Orthog. & 194.41 & 256.16 & 328.01 & 59.49 & 66.62 & 73.30 \\
\hline MKT & 0.11 & 0.28 & 0.44 & 0.12 & 0.87 & 2.15 \\
\hline SMB & 0.57 & 0.97 & 1.36 & 1.45 & 4.62 & 8.89 \\
\hline HML & 1.67 & 2.02 & 2.41 & 13.14 & 17.53 & 22.12 \\
\hline MOM & 1.30 & 1.55 & 1.80 & 16.89 & 23.40 & 30.50 \\
\hline Cov(SMB , HML) & & & & -5.29 & -3.55 & -1.90 \\
\hline Cov(HML , MOM) & & & & -10.59 & -8.94 & -7.31 \\
\hline Portfolio: & \multicolumn{6}{|c|}{$\mathrm{M}, \mathrm{S}, \beta, \sigma_{\epsilon} ; \gamma^{*}=3$} \\
\hline$\alpha /$ Orthog. & 80.16 & 118.27 & 162.00 & 40.88 & 46.15 & 52.06 \\
\hline MKT & 0.16 & 0.34 & 0.52 & 0.31 & 1.57 & 3.51 \\
\hline SMB & 0.92 & 1.23 & 1.55 & 5.22 & 8.83 & 12.97 \\
\hline HML & 1.88 & 2.18 & 2.49 & 20.35 & 25.14 & 29.75 \\
\hline MOM & 1.60 & 1.80 & 2.01 & 31.12 & 38.56 & 46.16 \\
\hline Cov(SMB , HML) & & & & -7.49 & -5.96 & -4.43 \\
\hline Cov(HML , MOM) & & & & -15.05 & -13.77 & -12.35 \\
\hline Portfolio: & \multicolumn{6}{|c|}{$\mathrm{M}, \mathrm{S}, \beta, \bar{r} ; \gamma^{*}=3$} \\
\hline$\alpha /$ Orthog. & 133.58 & 182.53 & 237.68 & 61.91 & 68.70 & 75.21 \\
\hline $\mathrm{MKT}$ & -0.03 & 0.14 & 0.30 & 0.00 & 0.32 & 1.18 \\
\hline SMB & 0.45 & 0.81 & 1.15 & 1.10 & 3.77 & 7.34 \\
\hline HML & 1.08 & 1.37 & 1.66 & 6.17 & 9.43 & 13.02 \\
\hline MOM & 1.32 & 1.53 & 1.74 & 20.29 & 26.73 & 33.55 \\
\hline Cov(HML , MOM) & & & & -8.44 & -7.00 & -5.55 \\
\hline
\end{tabular}




\section{Table 5 -Continued}

Decomposition of portfolio expected return and variance based on

Fama-French-Carhart Factors

\begin{tabular}{|c|c|c|c|c|c|c|}
\hline \multirow[t]{2}{*}{ Metric } & \multicolumn{3}{|c|}{$\begin{array}{c}\text { Resampled } \\
\text { Out of sample } \\
\text { regression coefficient }\end{array}$} & \multicolumn{3}{|c|}{$\begin{array}{c}\text { Resampled } \\
\text { Out of sample } \\
\text { variance component }\end{array}$} \\
\hline & $2.5 \%$ ile & Mean & $97.5 \%$ ile & $2.5 \%$ ile & Mean & $97.5 \%$ ile \\
\hline Portfolio: & \multicolumn{6}{|c|}{$\mathrm{M}, \mathrm{V}, \mathrm{S}, \beta, \bar{r}, \sigma_{\epsilon} ; \gamma^{*}=3$} \\
\hline$\alpha$ / Orthog. & 186.86 & 255.51 & 334.57 & 59.41 & 66.50 & 73.23 \\
\hline MKT & 0.51 & 0.76 & 1.02 & 2.52 & 5.87 & 10.33 \\
\hline $\mathrm{SMB}$ & 0.38 & 0.85 & 1.31 & 0.62 & 3.63 & 8.09 \\
\hline HML & 2.11 & 2.59 & 3.11 & 20.28 & 28.32 & 36.89 \\
\hline $\mathrm{MOM}$ & 0.85 & 1.16 & 1.47 & 7.05 & 12.97 & 19.76 \\
\hline Cov(MKT , HML) & & & & -8.38 & -6.12 & -4.02 \\
\hline Cov(HML , MOM) & & & & -10.53 & -8.40 & -6.21 \\
\hline Portfolio: & \multicolumn{6}{|c|}{$\mathrm{M}, \mathrm{V}, \beta, \bar{r}, \sigma_{\epsilon} ; \gamma^{*}=3$} \\
\hline$\alpha$ / Orthog. & 125.79 & 187.91 & 259.35 & 58.45 & 64.19 & 69.79 \\
\hline MKT & 1.25 & 1.44 & 1.64 & 18.42 & 24.99 & 31.97 \\
\hline SMB & -1.04 & -0.65 & -0.30 & 0.56 & 2.51 & 5.31 \\
\hline HML & 1.54 & 2.02 & 2.54 & 13.17 & 20.86 & 29.27 \\
\hline MOM & 0.61 & 0.89 & 1.18 & 4.17 & 9.43 & 15.99 \\
\hline Cov(MKT , HML) & & & & -13.17 & -10.94 & -8.68 \\
\hline Cov(HML , MOM) & & & & -8.21 & -6.09 & -4.06 \\
\hline Portfolio: & \multicolumn{6}{|c|}{$\mathrm{M}, \mathrm{S}, \bar{r}, \sigma_{\epsilon} ; \gamma^{*}=10$} \\
\hline$\alpha /$ Orthog. & 80.40 & 101.90 & 126.70 & 53.73 & 58.33 & 63.02 \\
\hline MKT & 0.50 & 0.56 & 0.62 & 13.26 & 18.51 & 24.66 \\
\hline SMB & -0.14 & -0.01 & 0.11 & 0.00 & 0.12 & 0.61 \\
\hline HML & 0.97 & 1.09 & 1.22 & 24.99 & 29.59 & 34.04 \\
\hline MOM & 0.51 & 0.59 & 0.68 & 14.83 & 19.55 & 24.60 \\
\hline Cov(MKT , HML) & & & & -12.99 & -11.27 & -9.58 \\
\hline Cov(HML , MOM) & & & & -12.13 & -10.64 & -9.16 \\
\hline Portfolio: & \multicolumn{6}{|c|}{$\mathrm{S}, \bar{r}, \sigma_{\epsilon} ; \gamma^{*}=10$} \\
\hline$\alpha /$ Orthog. & 98.89 & 118.35 & 140.22 & 55.29 & 59.97 & 64.77 \\
\hline $\mathrm{MKT}$ & 0.58 & 0.63 & 0.69 & 19.26 & 25.00 & 31.14 \\
\hline SMB & -0.25 & -0.13 & -0.02 & 0.01 & 0.64 & 1.83 \\
\hline HML & 0.85 & 0.96 & 1.07 & 20.29 & 24.20 & 28.24 \\
\hline MOM & -0.14 & -0.08 & -0.02 & 0.03 & 0.43 & 1.03 \\
\hline Cov(MKT , HML) & & & & -13.28 & -11.85 & -10.40 \\
\hline
\end{tabular}


Table 5 -Continued

Decomposition of portfolio expected return and variance based on

Fama-French-Carhart Factors

\begin{tabular}{|c|c|c|c|c|c|c|}
\hline \multirow[t]{2}{*}{ Metric } & \multicolumn{3}{|c|}{$\begin{array}{c}\text { Resampled } \\
\text { Out of sample } \\
\text { regression coefficient }\end{array}$} & \multicolumn{3}{|c|}{$\begin{array}{c}\text { Resampled } \\
\text { Out of sample } \\
\text { variance component }\end{array}$} \\
\hline & $2.5 \%$ ile & Mean & $97.5 \%$ ile & $2.5 \%$ ile & Mean & $97.5 \%$ ile \\
\hline Portfolio: & \multicolumn{6}{|c|}{$\mathrm{M}, \bar{r}, \sigma_{\epsilon} ; \gamma^{*}=10$} \\
\hline$\alpha /$ Orthog. & 43.95 & 60.46 & 78.48 & 27.75 & 32.85 & 38.36 \\
\hline MKT & 0.80 & 0.84 & 0.88 & 43.95 & 51.44 & 59.04 \\
\hline SMB & -0.94 & -0.84 & -0.76 & 20.95 & 24.19 & 27.49 \\
\hline HML & 0.28 & 0.34 & 0.42 & 2.38 & 3.71 & 5.20 \\
\hline MOM & 0.50 & 0.57 & 0.65 & 17.99 & 23.01 & 28.23 \\
\hline $\operatorname{Cov}(\mathrm{MKT}, \mathrm{SMB})$ & & & & -19.42 & -17.95 & -16.37 \\
\hline $\operatorname{Cov}(\mathrm{MKT}, \mathrm{HML})$ & & & & -8.05 & -6.65 & -5.26 \\
\hline $\operatorname{Cov}(\mathrm{MKT}, \mathrm{MOM})$ & & & & -8.38 & -7.47 & -6.50 \\
\hline Portfolio: & \multicolumn{6}{|c|}{$\mathrm{M}, \mathrm{S}, \sigma_{\epsilon} ; \gamma^{*}=10$} \\
\hline$\alpha /$ Orthog. & 47.18 & 60.10 & 74.05 & 44.90 & 48.00 & 51.02 \\
\hline MKT & 0.45 & 0.50 & 0.56 & 12.82 & 17.42 & 22.67 \\
\hline SMB & -0.03 & 0.07 & 0.17 & 0.00 & 0.24 & 0.92 \\
\hline HML & 1.06 & 1.16 & 1.26 & 35.23 & 38.90 & 42.44 \\
\hline MOM & 0.57 & 0.64 & 0.71 & 22.27 & 26.97 & 31.90 \\
\hline $\operatorname{Cov}(\mathrm{MKT}, \mathrm{HML})$ & & & & -14.15 & -12.54 & -10.91 \\
\hline Cov(HML , MOM $)$ & & & & -15.39 & -14.35 & -13.27 \\
\hline Portfolio: & \multicolumn{6}{|c|}{$\mathrm{M}, \mathrm{S}, \bar{r} ; \gamma^{*}=10$} \\
\hline$\alpha /$ Orthog. & 10.51 & 20.80 & 32.04 & 9.29 & 12.17 & 15.46 \\
\hline MKT & 1.09 & 1.12 & 1.15 & 82.81 & 87.19 & 91.25 \\
\hline SMB & -0.30 & -0.23 & -0.15 & 0.74 & 1.69 & 2.91 \\
\hline HML & -0.20 & -0.15 & -0.10 & 0.28 & 0.64 & 1.09 \\
\hline MOM & 0.23 & 0.29 & 0.35 & 3.64 & 5.61 & 7.83 \\
\hline $\operatorname{Cov}(\mathrm{MKT}, \mathrm{SMB})$ & & & & -8.11 & -6.10 & -4.08 \\
\hline Portfolio: & \multicolumn{6}{|c|}{$\mathrm{M}, \mathrm{V}, \mathrm{S}, \bar{r}, \sigma_{\epsilon} ; \gamma^{*}=10$} \\
\hline$\alpha /$ Orthog. & 78.26 & 99.65 & 123.90 & 49.34 & 54.85 & 60.28 \\
\hline MKT & 0.53 & 0.60 & 0.67 & 14.76 & 20.55 & 27.19 \\
\hline SMB & -0.21 & -0.07 & 0.07 & 0.00 & 0.25 & 1.10 \\
\hline HML & 1.08 & 1.23 & 1.38 & 29.64 & 36.19 & 42.64 \\
\hline MOM & 0.45 & 0.54 & 0.63 & 11.22 & 15.99 & 21.05 \\
\hline $\operatorname{Cov}(\mathrm{MKT}, \mathrm{HML})$ & & & & -15.54 & -13.14 & -10.86 \\
\hline Cov(HML , MOM $)$ & & & & -12.18 & -10.62 & -8.98 \\
\hline
\end{tabular}




\section{Table 5 -Continued}

Decomposition of portfolio expected return and variance based on

Fama-French-Carhart Factors

\begin{tabular}{|c|c|c|c|c|c|c|}
\hline \multirow[t]{2}{*}{ Metric } & \multicolumn{3}{|c|}{$\begin{array}{c}\text { Resampled } \\
\text { Out of sample } \\
\text { regression coefficient }\end{array}$} & \multicolumn{3}{|c|}{$\begin{array}{c}\text { Resampled } \\
\text { Out of sample } \\
\text { variance component }\end{array}$} \\
\hline & $2.5 \%$ ile & Mean & $97.5 \%$ ile & $2.5 \%$ ile & Mean & $97.5 \%$ ile \\
\hline Portfolio: & \multicolumn{6}{|c|}{$\mathrm{M}, \mathrm{S}, \beta, \bar{r}, \sigma_{\epsilon} ; \gamma^{*}=10$} \\
\hline$\alpha /$ Orthog. & 77.68 & 100.42 & 126.87 & 53.86 & 58.39 & 63.05 \\
\hline MKT & 0.49 & 0.56 & 0.63 & 13.41 & 18.51 & 24.45 \\
\hline SMB & -0.14 & -0.01 & 0.13 & 0.00 & 0.12 & 0.62 \\
\hline HML & 0.98 & 1.10 & 1.23 & 25.57 & 30.43 & 35.11 \\
\hline MOM & 0.48 & 0.57 & 0.66 & 13.68 & 18.53 & 23.86 \\
\hline Cov(MKT , HML) & & & & -13.14 & -11.42 & -9.74 \\
\hline Cov(HML , MOM) & & & & -12.07 & -10.50 & -8.88 \\
\hline Portfolio: & \multicolumn{6}{|c|}{$\sigma_{\epsilon} ; \gamma^{*}=10$} \\
\hline$\alpha$ / Orthog. & 22.43 & 29.94 & 37.44 & 19.07 & 21.27 & 23.55 \\
\hline MKT & 0.73 & 0.76 & 0.79 & 67.04 & 72.54 & 77.92 \\
\hline $\mathrm{SMB}$ & -0.70 & -0.66 & -0.62 & 22.77 & 25.45 & 28.18 \\
\hline HML & 0.39 & 0.42 & 0.46 & 8.05 & 9.59 & 11.28 \\
\hline MOM & -0.02 & 0.01 & 0.05 & 0.00 & 0.06 & 0.26 \\
\hline $\operatorname{Cov}(\mathrm{MKT}, \mathrm{SMB})$ & & & & -22.66 & -21.87 & -21.04 \\
\hline Cov(MKT , HML) & & & & -13.65 & -12.74 & -11.82 \\
\hline Portfolio: & \multicolumn{6}{|c|}{$\mathrm{M}, \mathrm{S}, \sigma_{\epsilon} ; \gamma^{*}=16$} \\
\hline$\alpha /$ Orthog. & 40.30 & 50.84 & 61.98 & 42.92 & 46.24 & 49.41 \\
\hline MKT & 0.54 & 0.58 & 0.63 & 26.01 & 32.44 & 39.66 \\
\hline SMB & -0.27 & -0.18 & -0.08 & 0.30 & 1.49 & 3.31 \\
\hline HML & 0.87 & 0.96 & 1.04 & 33.16 & 37.38 & 41.34 \\
\hline MOM & 0.36 & 0.42 & 0.48 & 12.08 & 16.26 & 20.60 \\
\hline Cov(MKT , HML) & & & & -18.04 & -16.79 & -15.42 \\
\hline Cov(HML , MOM) & & & & -12.32 & -10.91 & -9.32 \\
\hline Portfolio: & \multicolumn{6}{|c|}{$\mathrm{S}, \sigma_{\epsilon} ; \gamma^{*}=16$} \\
\hline$\alpha /$ Orthog. & 38.40 & 46.83 & 55.80 & 35.30 & 39.15 & 42.78 \\
\hline MKT & 0.59 & 0.63 & 0.67 & 38.55 & 46.13 & 54.18 \\
\hline SMB & -0.32 & -0.24 & -0.16 & 1.43 & 3.21 & 5.54 \\
\hline HML & 0.71 & 0.78 & 0.86 & 25.59 & 30.06 & 34.42 \\
\hline MOM & -0.07 & -0.03 & 0.00 & 0.00 & 0.17 & 0.58 \\
\hline $\operatorname{Cov}(\mathrm{MKT}, \mathrm{SMB})$ & & & & -8.58 & -6.14 & -3.91 \\
\hline Cov(MKT , HML) & & & & -18.84 & -17.96 & -17.02 \\
\hline
\end{tabular}


Table 5 -Continued

Decomposition of portfolio expected return and variance based on

Fama-French-Carhart Factors

\begin{tabular}{|c|c|c|c|c|c|c|c|}
\hline \multirow[t]{2}{*}{ Metric } & \multicolumn{3}{|c|}{$\begin{array}{c}\text { Resampled } \\
\text { Out of sample } \\
\text { regression coefficient }\end{array}$} & & \multicolumn{3}{|c|}{$\begin{array}{c}\text { Resampled } \\
\text { Out of sample } \\
\text { variance component }\end{array}$} \\
\hline & $2.5 \%$ ile & Mean & $97.5 \%$ ile & & $2.5 \%$ ile & Mean & $97.5 \%$ ile \\
\hline Portfolio: & \multicolumn{7}{|c|}{$\mathrm{M}, \sigma_{\epsilon} ; \gamma^{*}=16$} \\
\hline$\alpha /$ Orthog. & 15.90 & 23.43 & 31.27 & & 20.31 & 22.48 & 24.69 \\
\hline $\mathrm{MKT}$ & 0.74 & 0.76 & 0.79 & & 63.67 & 68.81 & 73.86 \\
\hline SMB & -0.72 & -0.67 & -0.63 & & 22.36 & 24.97 & 27.54 \\
\hline HML & 0.40 & 0.45 & 0.49 & & 8.37 & 10.09 & 11.92 \\
\hline MOM & 0.33 & 0.38 & 0.43 & & 12.80 & 16.68 & 20.83 \\
\hline $\operatorname{Cov}(\mathrm{MKT}, \mathrm{SMB})$ & & & & & -22.03 & -21.10 & -20.08 \\
\hline Cov(MKT , HML) & & & & & -13.73 & -12.72 & -11.65 \\
\hline Portfolio: & \multicolumn{7}{|c|}{$\mathrm{M}, \mathrm{S} ; \gamma^{*}=16$} \\
\hline$\alpha /$ Orthog. & -4.88 & 0.74 & 6.48 & & 4.26 & 5.17 & 6.21 \\
\hline MKT & 1.01 & 1.02 & 1.04 & & 100.60 & 102.16 & 103.56 \\
\hline SMB & -0.43 & -0.38 & -0.32 & & 4.76 & 6.44 & 8.25 \\
\hline HML & -0.06 & -0.03 & 0.01 & & 0.00 & 0.04 & 0.15 \\
\hline MOM & 0.09 & 0.14 & 0.18 & & 0.70 & 1.78 & 3.11 \\
\hline Cov(MKT , SMB) & & & & & -14.76 & -13.04 & -11.25 \\
\hline Portfolio: & \multicolumn{7}{|c|}{$\mathrm{M}, V_{-f}, \mathrm{~S}, \sigma_{\epsilon} ; \gamma^{*}=16$} \\
\hline$\alpha /$ Orthog. & 39.84 & 50.16 & 61.17 & & 36.12 & 40.02 & 44.01 \\
\hline MKT & 0.58 & 0.63 & 0.68 & & 29.48 & 36.34 & 43.75 \\
\hline SMB & -0.32 & -0.23 & -0.13 & & 0.77 & 2.30 & 4.40 \\
\hline HML & 0.98 & 1.07 & 1.17 & & 40.05 & 44.93 & 49.67 \\
\hline MOM & 0.30 & 0.36 & 0.42 & & 8.04 & 11.71 & 15.69 \\
\hline Cov(MKT , HML) & & & & & -21.10 & -19.49 & -17.75 \\
\hline Cov(HML , MOM $)$ & & & & & -11.79 & -10.14 & -8.36 \\
\hline Portfolio: & \multicolumn{7}{|c|}{$\mathrm{M}, \mathrm{S}, \beta, \bar{r}, \sigma_{\epsilon} ; \gamma^{*}=16$} \\
\hline$\alpha /$ Orthog. & 53.46 & 69.72 & 87.92 & & 48.43 & 52.29 & 56.22 \\
\hline $\mathrm{MKT}$ & 0.52 & 0.57 & 0.62 & & 22.93 & 28.86 & 35.32 \\
\hline SMB & -0.29 & -0.19 & -0.10 & & 0.39 & 1.67 & 3.50 \\
\hline HML & 0.84 & 0.94 & 1.03 & & 28.55 & 33.03 & 37.50 \\
\hline MOM & 0.34 & 0.41 & 0.47 & & 10.21 & 14.06 & 18.06 \\
\hline Cov(MKT , HML) & & & & & -16.39 & -14.89 & -13.35 \\
\hline Cov(HML , MOM) & & & & & -10.97 & -9.53 & -8.04 \\
\hline \multicolumn{8}{|c|}{ Panel B. Factor volatility / correlation matrix } \\
\hline & & MKT & SMB & HML & MOM & & $\begin{array}{l}\text { Mean } \\
\text { return }\end{array}$ \\
\hline & MKT & 4.41 & 0.25 & -0.24 & -0.11 & & 67.49 \\
\hline & SMB & & 3.01 & -0.20 & 0.06 & & 23.25 \\
\hline & HML & & & 2.88 & -0.22 & & 28.85 \\
\hline & MOM & & & & 4.32 & & 59.62 \\
\hline
\end{tabular}

